\title{
Zur Kenntnis der Thrombocyten des Salamander= blutes und ihres Verhaltens bei der Gerinnung. Von
}

Friedrich Meves in Kiel.

Hierzu Tafel XXII-XXV und sechs Textfiguren.

Die vorliegende Mitteilung handelt von den zuerst von Gol ubew (68) beschriebenen, weiter von Vulpian (77), Stricker (77), Hayem (79) und vielen anderen untersuchten Spindelzellen des Amphibienblutes, welchen Dekhuyzen (92) den Namen Thrombocyten beigelegt hat.

Bis in die neueste Zeit hinein geben zahlreiche Autoren irrtümlicher Weise an, dass diese Spindelzellen von v. Recklinghau sen entdeckt worden seien.

v. Recklinghausen (66) hat in Froschblut, welches er in geglühten Porzellanschälchen auffing und in ein grosses Glasgefäss mit feucht gehaltener täglich erneuerter Luft brachte, nach drei bis vier Tagen auf der abgesetzten Schicht der roten Blutkörperchen kleine weisse Punkte auftreten sehen, welche an den folgenden Tagen zu platten Inseln bis zu einem Durchmesser von $4 \mathrm{~mm}$ wuchsen und aus farblosen stark kontraktilen Zellen bestanden. Ausserdem fanden sich in diesen Inseln, weit zahlreicher aber in der unteren Serumschicht zerstreut, spindelförmige farblose Zellen, welche sich nach v. Recklinghausen vom vierten bis achten Tag zu roten Blutkörperchen weiter entwickeln.

Hayem (79, S. 609) und Ne umann (96, S. 247) haben nun aber bereits darauf hingewiesen, dass diese erst nach einigen Tagen auftretenden "Spindelzellen" mit den äusserst leicht sich verändernden Thrombocyten des zirkulierenden Blutes unmöglich identisch sein können. Auch mir scheint eine solche Identităt vollständig ausgeschlossen. Soweit ich (in Übereinstimmung mit Neumann) finden kann, ist Golubew, ein Schüler von Rollet, der erste, welcher die Spindelzellen als solche geseben hat. Er konnte sie nicht nur im frisch hergestellten Blutprăparat, sondern auch in den Kapillargefässen beobachten (68,S. 566-567). Golubew selbst glaubt allerdings 
auch irrtümlicher Weise, dass die Priorität seiner Entdeckung v. Recklinghausen zusteht.

Mit Hayem (79), Bizzozero (82) und vielen anderen und im Gegensatz zu Löwit (84), Neumann (96), A rnold (98), E. Schwalb e (00), Riess (04), Helber (05) nehme ich an, dass die Spindelzellen des Amphibienblutes die Analoga derjenigen Elemente des Säugetierblutes sind, welche Bizzozero (82) als Blutplättchen bezeichnet hat: ich bezweifle, dass diese letzteren Zerfallsprodukte von roten Blutkörperchen oder Leukocyten darstellen.

\section{Untersuchungsmethode.}

Die Spindelzellen zeichnen sich bekanntlich vor den anderen zelligen Elementen des Blutes dadurch aus, dass sie ausserhalb der Gefässe ausserordentlich rasch Verănderungen eingehen, welche man gewöhnlich als Zerfall bezeichnet. In einem frisch eingedeckten Tropfen vom Blut des Feuersalamanders (Salamandra maculosa) sind sie nur in den ersten Augenblicken nach Herstellung des Prăparates noch unverändert.

Wir wissen, dass diese Veränderungen sich durch Zusatz von sogenannten indifferenten Flüssigkeiten, wie Jodserum von M. Schultze und isotonischer Kochsalzlösung, eine zeitlang hintanhalten bezw. stark verlangsamen lassen ${ }^{\mathbf{1}}$ ). Ich habe $0,8 \%$ ige Kochsalzlösung ${ }^{2}$ ) verwandt, deren Zusatz ich in folgender Weise bewerkstellige. Ich schneide einem Salamander die Schwanzspitze ab und spüle den blutenden Stumpf kurz in einem Gefäss mit 0,8\% iger Kochsalzlösung ab. Die Lösung, welche der Wundfläche nach dem Herausziehen des Stumpfes anhaften bleibt, vermischt sich sofort mit dem vorquellenden Blut und wird mit diesem zusammen auf einen Objekttrăger abgetupft und eingedeckt.

1) Die Methodik der Kochsalzuntersuchung ist bekanntlich von Dekhuyzen soweit ansgebildet worden, dass es gelingt, sämtliche Blutzellen vom Frosch Wochen hindurch lebend zu erhalten. Herr Prof. Dekhuyzen hat mich im Herbst 1904 in Utrecht in seinem Laboratorium aufs freundlichste aufgenommen und mir die Kenntnis seiner Technik in liebenswürdigster Weise vermittelt; ich spreche ihm dafür meinen herzlichsten Dank aus.

2) Nach brieflicher Mitteilung von Dekhuyzen hat das Blut von Salamandra maculosa einen Gefrierpunkt von $-0,479^{\circ}$, ist demnach mit rund $0,8 \% \mathrm{NaCl}$ isotonisch. 
Um die Spindelzellen im unveränderten Zustand zu fixieren, kann man dem Blut verschiedene Mittel zusetzen. Haye m empfiehlt Pacinische Flüssigkeit und Osmiumsâure. Gute Fixierung und gleichzeitige Färbung kann man durch das von Dekhuyzen (92, S. 90) angegebene Osmium-Essigsăuregemisch erzielen, welches $6 \%$ kalt gesăttigte, wässerige Methylenblaulösung (und etwas Säurefuchsin) enthält; Fig. 16 ist nach einem mit diesem Gemisch behandelten Präparat gezeichnet.

An getrockneten Ausstrichpräparaten von Salamanderblut haben die Spindelzellen stets melrr oder weniger stark gelitten (vergl. auch Neumann 96, S. 252). Um Balsampräparate gut erhaltener Spindelzellen zu gewinnen, habe ich Blut auf dem Objektträger durch eine Schleuderbewegung ansgebreitet und diesen sofort in ein mit Fixierungsflüssigkeit (siehe unten) gefülltes Glas hineingebracht. Auf diese Weise sind die den Figg. 17, 29 und 30 zugrunde liegenden Präparate hergestellt. Die Veränderungen, welche an den Spindelzellen und in ihrer Umgebung im extravasierten Blut auftreten, habe ich teils an frischen, mit Paraffin umrandeten, teils an fixierten Präparaten studiert. Letztere gewinne ich in der Weise, dass ich etwas Blut in nicht zu dünner Schicht auf einem Objekttriger ausstreiche und diesen sofort in eine feuchte Kammer bringe, in welcher ich ihn verschieden lange Zeit (ein paar Minuten bis $z \mathfrak{u}$ mehreren Stunden) sich selbst überlasse. Dann stecke ich ihn in Fixierungsflüssigkeit, nachdem ich ihn vorher eventuell ein paar Mal in der Luft umbergeschwenkt habe, lamit die Ränder der geronnenen Blutschicht leicht antrocknen.

Im einzelnen habe ich zur Technik dieser Untersuchung noch folgendes $\mathrm{zu}$ bemerken:

In die feuchte Kammer bringe ich nicht Wasser, sondern $0,8 \%$ ige, mit dem Blut isotonische Kochsalzlösung, welche den gleichen Dampfdruck hat wie dieses. Nimmt man Wasser, so muss das Blut Wasserdampf anziehen und absorbieren. B öt tch er (Virchows Arch., Bd. 35, 1866, S. 128-129) teilt mit, dass die roten Blutkörperchen bei längerem Aufenthalt in der mit Wasser beschickten feuchten Kammer zerstört werden.

Als feuchte Kammer habe ich zuerst eine Glasglocke verwandt, welche innen mit Fliesspapier ausgeschlagen war. Da aber nach dem Wiederaufsetzen der abgehobenen Glasglocke 
immer einige Zeit vergehen muss, bis der Luftraum mit Feuchtigkeit gesăttigt ist, habe ich mich später auch folgender Einrichtung bedient. Ein Blechkasten (Textfigur a) von $14 \mathrm{~cm}$ Länge, $7^{1 / 2} \mathrm{~cm}$ Breite und $5^{1 / 2} \mathrm{~cm}$ Höhe trägt an einer der beiden langen Seitenwände eine dicke Metallplatte angelötet. Seitenwand und Metallplatte werden in halber Höhe des Kastens von einem horizontalen, $8^{1 / 2} \mathrm{~cm}$ langen, ca. $4 \mathrm{~mm}$ hohen Spalt durchsetzt. In diesen ist ein Metallbalken (mit Hilfe von Vaselin luftdicht) eingepasst, der eine horizontal stehende kreisförmige Scheibe

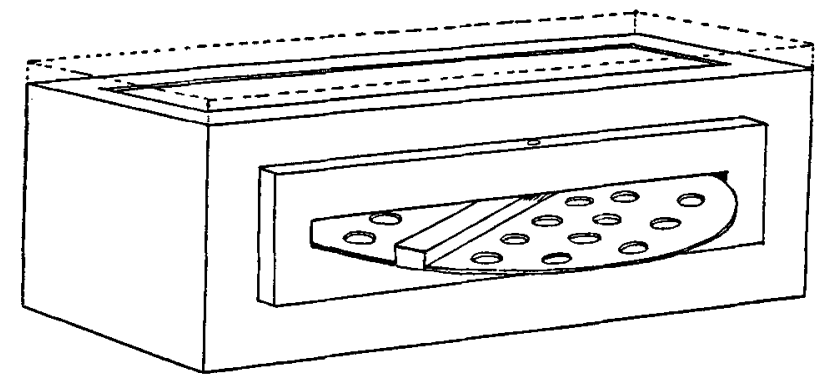

Fig. a.

trăgt. Metallbalken und Scheibe sind um eine durch ihre Mitte gehende senkrechte Achse drehbar. Der Kasten, welcher oben offen ist, wird, nachdem $0,8 \%$ ige Kochsalzlösung bis zu ca. $1 \mathrm{~cm}$ Höhe hineingefüllt ist, durch eine Glasplatte mit Hilfe von Vaselin luftdicht geschlossen und ist dann, sobald Sättigung im Innern eingetreten ist, zur Verwendung fertig. Man legt den mit frisch ausgestrichener Blutschicht bedeckten Objektträger (ich benutze solche von Giessener Format) auf die ausserhalb des Kastens befindliche Hülfte der Scheibe und bringt diese dann mitsamt dem Objektträger durch eine Drehung um $180^{\circ}$ in das dampfgesättigte Innere hinein. Die ganze Manipulation lässt sich sehr schnell vornebmen. Der Luftwechsel ist unter diesen Umständen sehr gering.

Als Fixierungsflüssigkeit habe ich vorwiegend entweder $1 \%$ ige Sublimatlösung oder Flemmingsche Chromosmiumessigsäure, letztere meistens in Form des sogenannten schwachen Gemisches ${ }^{1}$ ), beide mit Zusatz von $1 \%$ Kochsalz, benutzt.

1) $1 \%$ ige Chromsäure $25 \mathrm{ccm}, 1 \%$ ige Osmiumsäure $10 \mathrm{ccm}, 1 \%$ ige Essigsäure $10 \mathrm{ccm}$, dest. Wasser $55 \mathrm{ccm}$. 
Auf die Sublimatfixierung liess ich meistens eine Färbung mit Ehrlich-Biondischer Lösung folgen; nach Fixierung mit Flemmingschem Gemisch habe ich eine Doppelfärbung mit Safranin und verdünntem Delafield schen Haematoxylin oder die Fle m ming sche Dreifachbehandlung (Safranin-Gentiana-Orange) angewandt. ${ }^{1}$ )

Die mit Chromosmiumessigsäure fixierten Präparate übertrage ich nach der Färbung und Entwässerung in absolutem Alkohol aus diesem nicht sofort in Xylol, sondern vorber in ein Gemisch von absolutem Alkohol und Xylol zu gleichen Teilen; auf diese Weise werden Zerreissungen der fixierten Blutschicht, welche sonst auftreten, verhindert.

Ein Vergleich der Figuren auf Tafel 22, welche frische Objekte betreffen, mit den bei gleicher Vergrösserung nach Sublimatpräparaten gezeichneten Figuren 17 bis 28 auf Tafel 23 lehrt, dass die Sublimatlösung eine stark schrumpfende Wirkung ausübt, im übrigen aber den Naturzustand ziemlich getreu konserviert; während das Flemming sche Gemisch (Tafel 24 und 25) meistens eine Quellung der Protoplasmafortsatze bewirlit, die im extravasierten Blut an den Spindelzellen auftreten.

\section{Die unveränderten Thrombocyten.}

Form. Die unveränderten Thrombocyten (bezw. diejenigen des zirkulierenden Blutes) sind beim Salamander ebenso wie beim Frosch entweder spindelförmig oder auch keulenbezw. mandelförmig. Wenn man sie um ihre Längsachse rotieren sieht, bemerkt man, dass der Querdurchmesser abwechselnd länger und kürzer erscheint; woraus hervorgeht, dass sie auf einem Querschnitt abgeplattet sein müssen. Übereinstimmende Angaben bezüglich der Form der Froschspindelzellen finden sich bei Hayem (79, S. 203), Bizzozero (82, S. 325), Eberth (87, S. 41), Eberth und Schimmelbusch $(88$, S. 71),

1) Bei der Safranin-Haematoxylin-Doppelfärbung verfuhr ich in der Weise, dass ich die Präparate zuerst für ca. 24 Stunden in eine 1\% ige wässerige Safraninlösung hineinbrachte, dann mit neutralem Alkohol extrahierte und schliesslich ca. 6 bis 12 Stunden mit stark verdünntem Delafieldschen Haematoxylin nachfärbte. Die Flemmingsche Dreifachbehandlung habe ich im wesentlichen nach der von Flemming gegebenen Vorschrift ausgeführt. 
Dekhuyzen (92, S. 97), Neumann (96, S. 235), Giglio-Tos (98, S. 159).

Zellsubstanz. Die relativ spärlich vorhandene Zellsubstanz finde ich in Übereinstimmung mit den meisten Untersuchern und im Gegensatz zu Vulpian (77, S. 1280/81), Hayem (79, S. 241), und Marquis (92, S. 64-67) stets haemoglobinlos. Was ibren Bau anlangt, so ist sie nach einigen Autoren (z. B. Ne u mann 96, S. 242) gänzlich, nach anderen (H a y em (79, S. 206), Eberth (87, S. 41 und 44), Eberth und Schim melbusch (88, S. 7)), "fast" homogen. Bizzozero (82, S. 325) bezeichnet sie als feinkörnig, Dekbuyzen (92, S. 98) als „feinkörnig-feinfaserig mit konzentrischem Verlauf der Fibrillen." Nach Giglio-T os (98, S. 161) lässt sie an erhitzten Trockenpräparaten; welche mit Methylenblau BX gefärbt und in Glyzerin eingeschlossen sind, zwei Bestandteile erkennen, eine blau gefärbte Filarmasse und rötlich tingierte Granula, welche die Maschen der Filarmasse einehmen.

Ich selbst sehe an den lebenden, ohne jeden Zusatz untersuchten Zellen im Cytoplasma eine verwaschene Zeichnung, die ebenso gut einem feinkörnigen wie einem Fadenbau entsprechen könnte (Fig. 1-3). Nach Sublimatfixierung erscheint die Zellsubstanz homogen (Fig. 17). Auch an den mit Flem ming schem Gemisch behandelten Präparaten lässt sie von einem Fadenbau nichts erkennen, weist vielmehr ein undeutlich körniges Ausseben auf, welches ich in den Figg. 29 und 30 wiederzugeben versucht habe, und ist nicht selten an einem oder auch an beiden Polen des Kernes stärker verdichtet.

Das Cytoplasma der lebend untersuchten Zellen zeigt häufig stark lichtbrechende Kügelchen, welche meistens an den Polen des Kerns in năchster Nähe desselben liegen. Nach Ha y e m (79), welcher sie zuerst bei Rana temporaria aufgefunden hat, kommen bier ein bis vier derartige Kügelchen vor; bei Salamandra sind sie zuweilen noch zablreicher vorhanden.

Cytocentren. Eisen (97) und Giglio-Tos (98) hahen neuerdings "Centrosomen" im Cytoplasma der Spindelzellen beschrieben.

Hierzu möchte ich zunächst bemerken, dass meiner Meinung nach die Existenz von Centrosomen in den Spindelzellen über- 
haupt nicht in Frage kommen kann, sondern nur diejenige von Centriolen; vergl. hierzu: Fr. Meves, über die Frage, ob die Centrosomen Boveris als allgemeine und dauernde Zellorgane aufzufassen sind, Verh. d. anat. Ges. in Halle a. S., 1902.

Eis e n will "Centrosomen" bei Batrachoseps, einem in Kalifornien häufigen Batrachier, im allgemeinen an beiden Kernpolen gefunden haben; sie sind nach ihm hier von einer Reihe verschiedenartiger Cytoplasmahüllen umgeben.

Ich habe Gelegenheit gehabt, Präparate Eis ens einzusehen und zu konstatieren, dass sie mit den von ihm gegebenen Abbildungen in Übereinstimmung sind, habe aber keine Sicherheit gewinnen können, dass die fraglichen Körnchen Cytocentren bez. Centriolen darstellen. Wenn solche tatsăchlich, wie Eisen annimmt, an beiden Kernpolen vorkommen sollten, so müsste dies als auffallig bezeichnet werden; denn wir wissen, dass, wo sonst Centriolen in der ruhenden Zelle in grösserer Zahl vorhanden sind, sie sich meistens an einer Stelle zusammengruppieren $^{1}$ ).

Auch Giglio-Tos (98, S. 195 u f.) hat mich nicht davon zu überzeugen vermocht, dass er Centrosomen bezw. Centriolen vor sich gehabt hat. Diese präsentieren sich fast ausnabmslos als Doppelkörnchen; die von Giglio-Tos durch Methylenblaufärbung dargestellten „Centrosomen" der Spindelzellen dagegen sind in den meisten Fällen nur in der Einzahl vorhanden. Die von diesem Autor beobachtete schwache Strahlung aber kann als Beweis für die Cytocentrennatur der fraglichen Körnchen nicht verwertet werden, da eine ebensolche Strahlung sich nach ihm auch um Fremdkörper herum findet.

Ich selbst habe versucht mit Hilfe der Flemmingschen Dreifachbehandlung (Safranin-Gentiana-Orange) und der M. $\mathrm{Heiden}$ hainschen Eisenhämatoxylinmethode Centriolen in den Spindelzellen des Salamanders aufzufinden, habe aber bisher keinen Erfolg gehabt.

Plas mocyten. Eisen hat in seiner erwăhnten Arbeit (97) beschrieben, dass die polaren Teile des Protoplasmas (mit den in

1) Vergl. die Befunde über Centriolengruppen in Riesenzellen (M. Heidenhain, Arch. f. mikr. Anat. Bd. 43. 1894; Fr. Meves, Ebenda, Bd. 45, 1895; J. Broman, Anat. Anz., Bd. 17, 1900). 
ibnen enthaltenen "Centrosomen") sich ron den Spindelzellen abschnüren und neue morphologische Elemente bilden können, welche er als Plasmocyten bezeichnet.

Von diesen Plasmocyten hat Giglio-Tos anfänglich (97) behauptet, dass sie grösstenteils Spindelzellen, vielleicht auch junge Lymphocyten seien, deren Kerne sich infolge der von $\mathrm{E}$ i s e $\mathrm{n}$ angewandten Methode ${ }^{1}$ ) bis zur Unkenntlichkeit verändert hätten. Später aber (98), nachdem Eisen ihm Präparate übersandt hatte, hat er diese Kritik als irrtümlich zurückgezogen und die Plasmocyten für Körperchen besonderer Art erklärt, über deren Natur er hoffte sich demnächst bestimmt aussprechen zu können. Eine weitere Äusserung von Giglio-Tos zu diesem Gegenstand ișt aber meines Wissens nicht erfolgt.

Ich für meine Person muss es für ausgeschlossen erklären, dass solche Plasmocyten, wie sie $\mathrm{E}$ i s e $\mathrm{n}$ beschreibt, im zirkulierenden Salamanderblut vorkommen; dagegen dürften sie bei der Herstellung eines Blutpräparats auf artefiziellem Wege leicht folgendermassen entstehen können. Unmittelbar nachdem man einen frischen Bluttropfen eingedeckt bat, bemerkt man, wie bereits $\mathrm{Hayem}$ (79, S. 204) angibt, dass die Spindelzellen eine eigentümliche Viscositöt annehmen; „on les voit s'accrocher au verre, et comme ils sont très extensibles, le courant sanguin leur fait prendre, dès qu'ils sont fixés en un point, une forme allongée souvent désmesurée. " Besonders dann, wenn man einen Bluttropfen ausstreicht, wird es leicht passieren können, dass von einer solchen am Glas angeklebten Spindelzelle ein Stückchen Protoplasma abreisst. Jedenfalls finde ich in meinen Ausstrichpräparaten nicht selten kleine, völlig isolierte Protoplasmaklümpchen (ein solches ist in Fig. 41 nach Fixierung mit Fle m m in gschem Gemisch dargestellt), von denen ich auf Grund eines später zu schildernden Kennzeichens behaupten kann, dass sie höchstwahrscheinlich von einer Spindelzelle abstammen.

Kern. Die Form des Kerns wird übereinstimmend als länglich oval beschrieben. Nur Macallum (91, S. 242) gibt an, dass der Kern in den Spindelzellen von Necturus in einigen

1) Deckglaspräparate, die an der Luft 12 Stunden hindurch getrocknet und hierauf in Alkohol fixiert wurden; Färbung mit Toluidinblau, MethylenblauEosin und Ehrlich-Bi ondischer Lösung. 
Fällen gelappt sein kann; die Lappung kann soweit gehen, dass mehrere kleine kugelige Kerne vorhanden sind.

Was die Strukturverhältnisse des Thrombocytenkerns anlangt, so beschreibt $\mathrm{H}$ a y e $\mathrm{m}$ (79, S. 206), dass der Kern der in Jodserum untersuchten Spindelzellen des Froschbluts feine Körner zeigt, welche ziemlich regelmässig in der Weise angeordnet sind, dass der Anschein von Längsstreifen entsteht. Nach Fixierung in Sublimatgemischen konstatiert man (S. 208), dass die Körner peripher gelegen und kommaăhnlich sind; ibre Anordnung an derartigen Präparaten ruft bald den Eindruck einer Langsstreifung, bald denjenigen einer Querstreifung hervor.

Bizzozero (82, Taf. V, Fig. 14a) bildet zwei Spindelzellen des Frosches ab, deren Kern zwei parallel, dicht nebeneinander verlaufende Längslinien zeigt. Im Text habe ich eine auf die Längslinien bezügliche Bemerkung nicht finden können.

L öwit (83, S. 396 u. 85, S. 88) sagt, dass die Spindelzellen hinsichtlich der Struktur ihres Kernes vollständig mit den weissen Blutkörperchen übereinstimmen.

Nach Eberth (87, S. 43) beobachtet man an OsmiumsāurePräparaten an den Kernen der Spindeln mitunter einen leicht gewundenen Längsstreifen. „Wahrscheinlich rührt derselbe von einer Art Faltung der Kernwand oder geschrumpfter Kernsubstanz her." Der Kern lässt ausserdem einige glänzende Körner erkennen, von denen ein mehr rundliches wohl als Kernkörperchen anzusehen ist.

An gefărbten Trockenpräparaten zeigt der „länglich runde, nur da und dort leicht eingekerbte " Kern ein Chromatingerüst, welches unregelmässiger und schwächer ausgebildet ist als bei den farblosen Blutköperchen. Man erkennt nur 1-2 rundliche, intensiver gefärbte Körner, die Nucleolen und einige unregelmässige Klümpchen und Fädchen, die da und dort wohl zusammenhängen, aber nirgends ein so vollständiges Netzwerk bilden, wie man es an den Leukocyten und roten Blutscheiben findet.

Eberth und Schimmelbuscb (88, S. 77) erwahnen von dem Kern in den Spindelzellen von Triton cristatus, dass er sich bei Zusatz von verdünnter Essigsăure etwas authellt und dass an der Innenfläcbe seiner Wand ein glänzender Belag in Gestalt eines schmalen sichelförmigen Saums oder hellen Streifens erscheint. 
Mondino und Sala (88) und Mondino (ebenfalls 88) geben an, dass die Kerne der Spindelzellen sich mittels eines "kinetischen Prozesses" teilen, welcher, wenn auch von sehr eigentümlicher Art, einer Mitose ähnlich sei. Es scheint, dass diese Autoren die Längsstreifen von Hayem und Eberth, welche mit den gleich zu erwähnenden Mitochromen Dekhuyzens identisch sind. für Chromosomen angesehen haben.

Nach H. F. Müller (89, S. 32) zeigen die Kerne der Spindelzellen in: ihrem Innern ein System feiner, stellenweise etwas dickerer, feinzackig begrenzter Stränge als ein feinstes auch bei der stärksten Vergrösserung nicht sicher aufzulösendes Netzwerk. Die Stränge und das Netz färben sich in Safranin; bei weniger starken Vergrösserungen sieht man die Substanz. zwischen den Strängen in toto leicht rot gefärbt, was nur der Feinheit des Netzes zuzuschreiben ist, während bei starken Vergrösserungen die Kerngrandsubstanz deutlich ungefärbt erscheint. So verhält es sich immer an gut gelungenen Präparaten. Gröbere Anhäufungen von Chromatin in Form vom Klumpen sieht man nur, wenn veränderte stark gequollene Kerne vorliegen.

Luzet (91) findet bei der Taube im Kern der Spindelzellen ein Chromatinnetz, dessen Fäden regelmässige rautenförmige Maschen begrenzen. Die Knotenpunkte, von denen die Fäden ausgehen, sind ziemlich voluminös; die Fäden verdünnen sich bis zn ihrer Mitte, um dann wieder bis zum benachbarten Knotenpunkt an Dicke zuzunehmen.

Nach Macallum (91 S. 242) ist der Kern der Spindelzellen von Necturus entweder homogen oder grob reticuliert

Dekhuyzen (92, S. 98) beschreibt an den Spindelzellen des Froschbluts als am meisten charakteristisches Merkmal ( Leitmerkmal“) die von ihm sog. Mitochromen, streifen- und schleifenförmige Chromatinansammlungen, welche gewöhnlich in der Langsdimension des Kerns verlaufen. Sie scheinen zu der Membran in Beziehung zu stehen, insofern sie derselben anzuliegen pflegen. „Öfters verlaufen zwei Mitochromen über längere Strecken parallel und weichen auf einmal auseinander, indem sich ein neues schleifenförmiges Mitochrom in die von den beiden gebildeten Winkel einschiebt, welches dann wieder parallel verlauft mit den Enden der beiden ersten." "Es sieht so aus, "făhrt D ekhuyzen fort, „als ob auf dem Kern eine sich verzweigende Rinne vorhanden ist, welche von Chromatinfäden begrenzt wird." Man findet nach Dekbuyzen Bilder, welche zu Gunsten der Auffassung sprechen, dass es sich bei den Mitochromen um Chromosomen handelt, welche im ruhenden Kern persistieren, d. h. kontrahiert geblieben sind.

Ne umann (96, S. 241) hălt den Längsstreifen an den Kernen der Spindelzellen des Froschblutes für einen durch seine 
Dicke ausgezeichneten Balken eines im Innern des Kerns befindlichen Chromatingerüstes; Präparate aus $\mathrm{Pa}$ cin $\mathrm{i}-\mathrm{H}$ a y e m scher Flüssigkeit, mit einer essigsauren Vesuvinlösung gefärbt, liessen erkennen, dass von dem axialen (übrigens meist nach dem Rande des Kerns verschobenen) Balken zarte Chromatinfäden ausstrahlen, die mehrfach sich untereinander verbindend ein feines Netzwerk mit schmalen langen Maschen bilden; im Verlaufe dieser Fäden und an den Knotenpunkten des Netzes sind Verdickungen kaum wahrnehmbar; der erwähnte dickere Längsbalken, der zuweilen noch aus zwei parallelen,'nebeneinander gelagerten Fäden zusammengesetzt erschien, und die ebenfalls longitudinal ausgezogenen Maschen geben dem Retikulum ein einigermaßen charakteristisches Aussehen anderen Kernformationen gegenüber. "Am meisten" sagt $\mathrm{Ne} \mathrm{u} \mathrm{mann,} \mathrm{,dürfte} \mathrm{die} \mathrm{von} \mathrm{Dek} \mathrm{h} \mathrm{u} \mathrm{y} \mathrm{z} \mathrm{en} \mathrm{gegebene} \mathrm{Beschreibung}$ mit meiner Beobachtung zusammentreffen, weniger die Angaben von Luzet, Löwit und H. F. M üller."

Nach Giglio-To s (98, S 154-155) sind die Mitochromen Chromatinmassen von Strang- oder Bandform, welche in der Mehrzahl der Fălle mit unregelmăssigen Verdickungen besetzt sind; sie durchziehen den Kern der ausgewachsenen Thrombocyten entweder in der Lăngsrichtung oder schräg, zuweilen, aber nur selten, auch quer. Häufig sind zwei solcher Chromatinstränge vorhanden, welche parallel mit einander verlaufen; die inneren Ränder beider Stränge sind stets sehr deutlich und durch eine dazwischen eingelagerte schmale Schicht von Kernsaft getrennt, welche gewöhnlich schwächer gefärbt ist als der Rest und deshalb heller erscheint. Es gibt Falle, in denen diese Anordnung so stark in die Augen springt, dass es auf den ersten Blick scheint, als sei der Kern in zwei Teile geteilt; jedoch überzeugt man sich leicht davon, dass dies eben nur Schein ist.

Andere Male sind die Mitochromen nicht so lang und regermässig, aber zahlreicher und kürzer und deshalb weniger auffallend. Dann sind sie häufig ganz schräg oder fast quer angeordnet.

Nicht alles Kernchromatin wird durch diese charakteristischen Mitochromen repräsentiert. Daneben sind nach Giglio-Tos immer noch andere, im allgemeinen grosse Chromatinmassen vorhanden, die vom Kernsaft manchmal nur wenig abstechen, weil auch dieser sich ziemlich stark färbt; sie sind entweder rund oder 
von unregelmässiger Form, entweder im Kern verstreut oder der Membran desselben angelagert, einander benachbart oder miteinander verschmolzen. Viele von ihnen sind durch dicke Chromatinfaden unter einander oder auch mit den Mitochromen verbunden oder auch den letzteren so stark genähert, dass sie mit ihnen verschmelzen.

Was die Bedeutung der Mitochromen anlangt, so hält Giglio-Tos sie nicht für Chromosomen (M o nd in o und Sala, Dekbuyzen), sondern meint, dass es sich um eine besondere Anordnungsweise des Chromatins handelt, welche unter unbekanntem Einfluss zustande kommt.

Meine eigenen Beobachtungen an den durch ihre Grösse ausgezeichneten Thrombocyten des Salamanders haben mir ergeben, dass die sogenannten Mitochromen nicht, wie Dekhuyzen, Neumann und Giglio-Tos annehmen, im Innern des Kerns verlaufende Chromatinstränge sind. Es handelt sich vielmehr um spalt förmige Einsenkungen der Kernmembran, wie bei Salamandra schon am frischen Präparat (Fig. 1-3) $z u$ sehen ist.

Fixierte und gefärbte Präparate zeigen, dass die Innenseite der Kerumembran mit einer fast ununterbrochenen Chromatinlage bedeckt ist (vergleiche auch die oben zitierte Beobachtung, welche Eberth und Schimmelbusch bei Zusatz von verdünnter Essigsaure gemacht haben). Daher erhalt man, wenn man auf eine spaltförmige Einsenkung der Kernmembran einstellt, das Bild eines Chromatinbalkens, der aus zwei Parallelfaden zusammengesetzt erscheint (Fig. 16, 17, 29, 30). Der Zwischenraum zwischen den beiden Fäden wird nicht, wie Giglio-Tos meint, von Kernsaft, sondern von Zellsubstanz eingenommen; dass er am gefärbten Präparat heller erscheint als das Kerninnere, kann demnach nicht wunder nehmen.

Ähnliche spaltförmige Einfaltungen der Oberfläche sind hei anderen Kernen vielfach beobachtet. Flemming ${ }^{1}$ ) hat sie an den Kernen des Epithels und der Leydigschen Drüsenzellen in der Haut der Salamanderlarve beschrieben, ich selbst ${ }^{2}$ ) fand

1) W. Fl e m ming: Zellsubstanz, Kern und Zellteilung Leipzig 1882, S.95.

2) Fr. Meves: Über eine Metamorphose der Attraktionssphäre in den Spermatogonien ron Salamandra maculosa. Arch. f. mikr.Anat.Bd.44, 1895, S. 164. 
sie an den Kernen der Spermatogonien und der sogenannten Follikelzellen im Hoden und Ovarium des erwachsenen Tieres; bekannt ist ferner ihr Vorkommen an den Kernen der S e r tolischen Zellen des Saugetierhodens.

Was über den Verlauf der Einfaltungen am Kern der Spindelzellen zu sagen ist, deckt sich im wesentlichen mit dem, was Dekhuyzen und Giglio-Tos mit Bezug auf die ,Mitochromen" beschrieben haben; ihre Zah l scheint bei Salamandra meistens grösser za sein als bei Rana.

Im Innern des Thrombocytenkerns finde ich an den fixierten und gefärbten Präparaten keine gröberen Chromatinbrocken, wie Giglio-Tos, sondern zahlreiche feine Chromatinkörnchen; von diesen wird man auf Grund unserer sonstigen Kenntnisse vom Bau des Kerns annehmen dürfen, dass sie in den Străngen eines (an meinen Präparaten nicht zur Darstellung gebrachten) Liningerüstes gelegen sind.

Von dem Hay emschen ,Längsstreifen" am Kern der Froschspindelzellen hat Eberth bereits 1888 ausgesprochen, dass er wahrscheinlich ,von einer Art Faltung der Kernwand oder geschrumpfter Kernsubstanz" herrühre. In diesen Worten scheint allerdings zu liegen, was sicher nicht zutreffend ist, dass es sich nach Eberths Meinung um ein Kunstprodukt handelt. - Auch Dekhuyzen ist stellenweise nahe daran gewesen, die Natur der „Mitochromen" richtig zu erkennen, wie einige oben reproduzierte Wendungen seiner Beschreibung zeigen.

Nucleolen sind in der Regel nicht zu sehen, können aber, wie Hayem $(79$, S. 210$)$ bereits erwähnt, durch Osmiumsäure dargestellt werden; diese ist bekanntlich ein sehr geeignetes Mittel für den Nachweis von Nucleolen (vergl. W. F l e m min g, Zellsubstanz, Kern und Zellteilung, 1882, S. 141). Die Annahme Ne umanns (96, S. 241), dass die bei der Einwirkung der Osmiumsäure auftretenden Nucleolen Kunstprodukte seien, kann ich nicht für gerechtfertigt halten.

\section{Gestaltsveränderungen der Thrombocyten im extra- vasierten Blut.}

Grolubew (68, S. 568) konstatiert bereits, dass die Spindelzellen, die aus frisch abgelassenen Blut gewonnen sind, kürzer und dicker werden und sich zuletzt in Kugeln verwandeln, von 
denen er sagt, dass sie sich garnicht von den sogenannten „freien Kernen" unterscheiden. Einige von diesen Kugeln bekommen später einen hyalinen Hof.

Die erste eingehende Schilderung von dem Verhalten der Spindelzellen im extravasierten Blut von Frosch und Triton hat Stri cker (77, S. 10 u. folg.) gegeben. Die folgende Darstellung bezieht sich auf Triton. Stricker schreibt: „Da der Leib der ... Zellen amöboid ist, so variiert seine aussere Begrenzung, wie auch die Verteilung seiner Masse rings um den Kern. Nicht selten scheint diese auf viele Zacken oder Höcker, oder auf einige ăusserst dünne wie zerrissen aussehende Anhängsel reduziert zu sein. Andere male scheint der Kern in einem Teile seiner Zirkumferenz nackt und nur an einer oder der anderen Stelle mit einem aufgelagerten Klümpchen Protoplasma versehen zu sein. Wahrend der Zelleib seine Veränderungen durchmacht, bleibt indessen auch der Kern nicht ruhig. Er ändert seine Gestalt; er ist bald kugelig, bald elliptisch, bald wieder unregelmässig geformt. Vollends sein inneres Gerüste ist an ganz frischen Präparaten in einer ununterbrochenen Bewegung begriffen."

Das weitere Verhalten des Kerns führt Stricker zu einem Resultat, welches er besonders durch seine Beobachtungen an Leukocyten bestätigt findet: dass der Kern kein persistentes Formelement, sondern ein vorübergehend abgekapselter Teil des Zelleibes sei.

Wenn man sorgfältig auf die Konturen der Kernhülle achtet, so merkt man nach Stricker, dass sie ab und $\mathrm{zu}$ in gewissen Ebenen unterbrochen ist, und das Innengerüste kontinuierlich in den Zelleib übergeht.

Bei weiterer Beobachtang wird dieses Verhältnis noch prägnanter. „Die Kernhülle wird bald in einer grösseren Ausdehnung durchbrochen, und nunmehr stehen Kerngerüste und Zelleib in offener Verbindung. Die Kernhülle ist auf ein Drittel oder auf die Hälfte ihres früheren Umfanges reduziert und sitzt jetzt eigentlich nur wie eine unvollständige Kapsel auf einem amöboiden Klümpchen, etwa wie das Schneckenhaus auf der frei herumkriechenden Schnecke.

In der Mehrzahl der Fälle gestaltet sich das Verhältnis so, dass die Kernhülle in zwei oder mehr Stücke zerreisst, zumeist in ein grösseres und ein kleineres Stück, sodass der bewegliche Zelleib zwei ungleich grosse Kapselhälften trägt, etwa wie wenn die kriechende Schnecke auch am Kopfe ein Hütchen aufhätte". 
Ebensolche Zellen wie diejenigen, an welchen siricker die geschilderten Veränderungen beobachtet hat, kizimen nach ihm auch in Gruppen vor. Der Unterschied zwiscen beiden besteht nur darin, dass hier je ein Kern und dort je riele Kerne von einem Zelleibe umgeben zu sein scheinen.

Namentlich am Froschblut will Stricker sich sinliesslich überzeugt haben, dass die Protoplasmazone der kugelig sewordenen Spindelzellen verschwinden kann, unter Umständen. die keine andere Annahme zulassen, als dass das Protoplasma sich in das Innere des Kerns zurückgezogen hat; die Spindelzeilen haben sich in nackte Kerne umgewandelt.

Ha y e m (79) schildert (S. 204) die Neigung der Spindelzellen am Glas und aneinander anzukleben und beschreibt dann (S. 215 u. folg.) die Veränderungen, welche im Blut ron Rana viridis an den Zellen eines Spindelhaufens vor sich geben, der durch Verschmelzung von zirka zwölf Zellen gebildet ist.

Die Oberflache der Spindeln wird stachelich infolge einer Art Fältelung der Oberfläche und infolge der Bildung zallreicher kurzer Fortsätze. Bald scheinen die Zellen gegen einander anzudrängen, sie werden polyedrisch; der Haufen gleicht einer Art vielkerniger Platte oder einer Gruppe von Pflasterepithelien, die mit Zacken oder Stacheln versehen sind. Ausser den Fortsätzen, deren Anordnung von einem Augenblick zum andern wechselt, sieht man am Rande des Haufens durchsichtige Bläschen auftreten, welche erblassen und dann verschwinden; sie scheinen anzuzeigen, dass ein Teil des Protoplasmas sich in der Blutflüssiglieit löst.

Diese ersten Veränderungen haben zur Folge. dass das Protoplasma der Spindelzellen durchscheinender wird und der Kern sehr deutlich hervortritt. Später suchen die Zellen sich immer inniger aneinander zu lagern, sodass sie nach Ablauf einer verschieden langen Zeit (einige Minuten bis zu einer halben Stunde) eine einzige verschwommene Masse bilden. Am Rand dieser Massen bemerkt man protoplasmatische Verlängerungen, ferner Knospen, welche sich gewissermassen durch Segmentierung von der Hauptmasse abtrennen, und schliesslich mehr oder weniger voluminöse Bläschen. Die Kerne, welche zuerst sehr deutlich sind, werden, nachdem sie sich deformiert haben, trübe und leicht grau aussehen; eine Anzahl von ihnen fragmentiert sich, einige verschwinden sogar ganz. 
Nach Ablauf von $1 / 2-3 / 4$ Stunden, zuweilen früher, treten in den Haufen stark lichtbrechende Körperchen auf, welche zuerst wenig zahlreich sind, in der Folge aber immer reichlicher werden. Einige von ihnen zeigen ein zentrales Loch, sind also ringförmig; andere werden von mehreren Löchern (von sehr variablem, zuweilen sehr kleinem Durchmesser) durchbohrt. Man überzeugt sich leicht, dass sie von dem Kern der Spindelzellen abstammen. Eine Anzahl der Spindelzellen, deren Protoplasma geschwunden oder ausserst durchsichtig geworden ist, scheint um diese Zeit bereits untergegangen $\mathrm{zu}$ sein; andere haben sich $\mathrm{zu}$ blassen, schlecht begrenzten Platten umgeformt.

Nach Ablauf von $1 / \frac{1}{2}-2$ Stunden hat die Desorganisation des Spindelzellhaufens bereits grosse Fortschritte gemacht.

Die eben referierte Beschreibung ergänzt $\mathrm{Hay}$ em durch eine weitere ähnliche, welche das Verhalten eines Spindelzellhaufens im Blut von Rana temporaria betrifft, um schliesslich noch das Verhalten isolierter Spindelzellen desselben Tieres. zu schildern.

Eine isolierte Spindelzelle sieht man bei einer über mehrere Stunden ausgedehnten Beobachtung sich unaufhörlich unter Produktion von hellen Knospen und Bläschen umgestalten. Der Kern scheint komprimiert zu werden; oft sieht er wie eingeschnürt aus, scheint sich zu teilen oder blasigen Bildungen Platz zu machen; oder auch er ist an das eine Ende des Körperchens. zurückgedrängt und erscheint völlig frei; aber dies ist nur Schein ; nach Ablauf einiger Winuten findet er sich häufig von neuem im Innern der Zelle. Schliesslich erblasst die Spindelzelle mehr und mehr, wird unregelmässig, eckig und rerschwommen.

Hayem sagt: „D'après les modifications successives que présente chaque hématoblaste isolé, on pourrait croire qu'il s'agit d'un corpuscule poreux qui reviendrait sur lui-même et ferait sortir, par exosmose, une sorte de produit muqueux, tandis que le noyau comprimé, étranglé, parfois fragmenté, serait mis en quelque sorte en liberté. Mais ces apparences peuvent être interpretées également dans le sens de déformations compliquées ayant pour siége le disque même de l'hématoblaste dont une partie s'étalerait en plaque mince, colloidale et festonnée."

Die folgenden Untersucher, Bizzozero, Eberth, Eberth und Schimmelbusch, Macallum, Neumann, Deetjen, 
geben von den Veränderungen der Spindelzellen Darstellungen, welche im wesentlichen mit derjenigen $\mathrm{Hayems}$ übereinstimmen.

Bizzozero (82, S. 326) beschreibt, dass die Spindelzellen wenige Augenblicke nach der Herstellung des Präparates verkleinert erscheinen; an ihrer Oberfläche treten mehrére kleine Halbkugeln hyaliner Substanz auf oder werden dünne Lamellen gleichfalls hyaliner Substanz ausgeschieden; die Form des Kerns wird mehr eine rundliche. Während dieser Alteration werden die Zellen sehr viscös und haften daher leicht an fremden Körpern, z. B. an dem Deckgläschen, oder sammeln sich zu grossen Haufen, in welchen sie dieselben Veränderungen wie im isolierten Zustand erleiden und mit einander verschmelzen. Wir haben dann eine Masse vor uns, die aus zahlreichen, aber undeutlichen Kernen und einer Substanz von körnigem Aussehen besteht, worin die Kerne eingebettet sind und welche an ihrer Peripherie nicht selten Tröpfchen oder Lamellen von körniger Substanz vorragen lässt.

Nach Eberth (87, S. 43) bestehen die Veränderungen, welche eine Spindelzelle zwischen Objektträger und Deckgläschen eingeht, zunächst darin, dass Protoplasma und Kern aufquellen; der früher glatte Kontur wird unregelmässig, das Körperchen erscheint wie gezackt, sein äusserer Kontur verschwommen; das Protoplasma, wohl infolge der starken Quellung des Kerns reduziert, lässt alsbald an der Oberfläche eine Menge kleiner, leicht gestielter Körnchen erkennen, die sich alsbald ablösen, sodass der Zellkörper in eine Menge kleiner Körnchen sich aufzulösen scheint, bis schliesslich nur noch ein matter, etwas unregelmässiger Fleck, der Kern der Spindel, übrig bleibt.

In ganz ähnlicher Weise wird das Verhalten isolierter Spindelzellen von Eberth und Schimmelbusch $(88, \mathrm{~S} .72)$ beschrieben. Dann heisst es weiter: „Liegen, wie das am häufigsten der Fall, drei, vier oder ein ganzes Häufchen von Spindeln zusammen, so verschmilzt das Protoplasma zu einer hellen bis feinkörnigen Masse. Die Kerne sind in dieser zunächst noch gut zu erkennen, und es dauert viel länger, bis sie einem erst grobkörnigen, später feinkörnigen Zerfall unterliegen."

Nach Macallum (91, S. 242) ist das Protoplasma der Spindeizellen von Necturus amöboid oder in Form feiner gerader Strahlen vorstreckbar, welche bei sorgfältiger Beobachtung zuweilen langsame vibrierende Bewegung erkennen lassen. In einer feuchten Kammer werden die vorher ,reticulierten Kerne homogen oder aber das Kernchromatin bildet eine dicke Schicht unter der kugelig gewordenen Kernmembran. Nunmehr kann ein Lappigwerden des Kerns eintreten (nach S. 244). Schliesslich zerlegt sich das ganze Körperchen in eine Anzahl von Kügelchen, deren Cytoplasmateile sich grösstenteils im Serum lösen.

Neumann (96, S. 244) konnte folgendes mit Sicherheit beobachten:

Das spindelförmige Körperchen zieht sich unter Verkürzung seines Längendurchmessers sehr bald zu einem kugligen oder etwas unregelmässigen Klümpchen zusammen und wird dabei zugleich etwas stärker glänzend; sein Aussehen gewinnt dadurch so sehr Ähnlichkeit mit den kleinsten, stets gleichzeitig im Präparate vorhandenen Leukocyten, dass es sehr schwierig oder selbst unmöglich ist, beide nunmehr zu unterscheiden. 
Bereits nach einigen Minuten erscheint das Körperchen blasser und weniger scharf konturiert, nach kurzer Zeit treten sodann die Umrisse eines runden, etwas granulierten Kernes hervor und die umgebende Substanz erscheint als eine spärliche, hyaline Umhüllung des Kerns, an deren Peripherie kleinste, tropfenartige, ebenfalls hyaline Gebilde haften; auf dem wechselnden Spiel dieser den Rand bildenden Tröpfchen, die immer schnell wieder verschwinden und durch andere ersetzt werden, beruht die beständige Formänderung des Körperchen und die bald zentrale bald exzentrische Lage des Kerns, an welchem letzteren $\mathrm{Neumann}$ auch Einschnürungen gesehen hat. Ein vollständiges Freiwerden der Kerne (Stricker, Hayem) hat er nicht konstatieren können.

Dass ein Zerfall der Kerne in den Zellen der Spindelhaufen zu Stande kommt, wie Eberth und Schimmelbusch angegeben haben, wird von Neumann in Abrede gestellt.

Nach De etjen (00) verlieren die Spindeln des Frosches, frisch untersucht, rasch ihre ovale Gestalt, indem sie rundlicher werden, und indem zugleich das Protoplasma, das anfangs scharf nach aussen abgegrenzt schien, jetzt in Form eines zarten Saumes mit unregelmässigen Rändern und kurzen Fortsätzen den grossen Kern umgibt. In dieser Form erhalten . sich die Zellen meist einige Zeit, äusserlich wenig verändert, nur wird das Plasma blasser, der Kern glänzender nnd seine feine Zeichnung undeutlicher, ein Zeichen der fortschreitenden Degeneration. Nach etwa einer Stunde, oft auch schon früher, ist das Aussehen der Spindeln aber schon wesentlich anders, Oberflächlich betrachtet sieht man nur einen ganz homogenen glänzenden Körper, den Kernrest. Das Protoplasma ist erst bei genauerem Zusehen als eine zarte, von einem überaus feinem Kontur begrenzte, gequollene Scheibe, welche diese Kernsubstanz umgibt, zu erkennen. Einzelne Kernpartikelchen findet man bisweilen losgebröckelt vom Kern in diesem Protoplasma liegen.

Dekhuyzen (92 und 01) Deetjen (00) und Zieler (03) haben Veränderungen beschrieben, welche unter Bedingungen vor sich gingen, die geschaffen waren, um die Spindelzellen längere Zeit lebend $z u$ erhalten.

Dekhuyzen (92) hat an Spindelzellen, welche in $0,8^{\circ}$; iger Kochsalzlösung suspendiert waren, amöboide Bewegungen wahrgenommen.

Wenn Deetjen (00) Froschblut auf eine Agarschicht brachte, die Kochsalz und metaphosphorsaures Natron enthielt, beobachtete er an den Spindelzellen die gleichen Veränderungen, wie am frisch untersuchten Blut; jedoch behielt der Kern für lange Zeit seine „zarte" Struktur und wurde nicht zu einer homogenen Masse umgewandelt; was nach $D$ eetjen beweist, dass die Degeneration der Spindeln durch metaphosphorsaures Natron aufgehalten wird.

Dekhuyzen (01, S. 537) schildert ,agonale Erscheinungen“, die an den Spindelzellen des Frosches unter dem Einfluss einer 0,8\% igen, besser einer hypotonischen Kochsalzlösung auftreten. 
Fängt man Froschblut im Becherglas in 0,5"/oiger Kochsalzlösung auf und breitet einen Tropfen vom Bodensatze auf dem Objektträger aus, so fangen bald einige Spindelzellen an, eine Reihe von Veränderungen zu erleiden, welche in 3 bis 5 Minuten ablaufen. Im Bechergliischen erhalten sie sich länger. Als Reiz, welcher die Agone in Gang bringt, können der Transport in der Pipette und die unge-

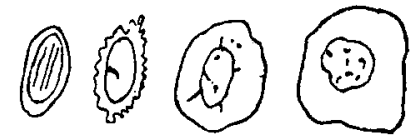

Fig. b.

"Agone" eines Thrombocyten des

Frosches in $0,5 \%$ igem $\mathrm{NaCl}$. Nach Dekhuyzen (01 S. 537 .). wohnten Bedingungen (z. B. Erhitzung, weil stets bei Gas mikroskopiert wurde) unter dem Deckglase in Betracht kommen. Eine glattrandige, ovale Spindelzelle fängt plötzlich an, sich zu kontrahieren, wird kleiner und mehr kugelig, dabei wird die Oberfläche höckerig, das Plasma grünglänzend, d. h. stärker lichtbrechend, dann erscheint, etwa 80 Sekunden nach den ersten Veränderungen, ein äusserst blasskonturierter "Halo," der sich schnell ausdehnt und innerhalb dessen das den Kern umgebende „Plasma" Gestaltsveränderungen erleidet.

Zieler (03) hat die von Dekhuyzen 1892 erwähnten ,amöboiden Veränderungen", welche die Spindelzellen in $0,8 \%$ iger Kochsalzlösung zeigen, in detaillierterer Weise beschrieben; er fast dieselben als "spezifische $\mathrm{Ab}$ sterbeerscheinungen" auf.

Meine eigenen Beobachtungen über die Veränderungen extravasierter Spindelzellen des Salamanders habe ich an frischem, ohne Zusatze eingedecktem Blut angestellt und durch fixierte Präparate, welche in der oben (S. 313-315) geschilderten Weise gewonnen waren, kontrolliert.

Die Figg. 4a-f stellen sechs aufeinander folgende Stadien der Veränderung dar, welche an ein und derselben lebenden Zelle beobachtet sind. Auf dem zuerst gezeichneten Stadium 4 a (fast unmittelbar nach Herstellung des Präparates) haben Zelleib und Kern sich bereits im Längsdurchmesser verkürzt. Diese Verkürzung geht sofort weiter. Die Zelloberfläche bedeckt sich mit zahlreichen kleinen halbkugeligen Vorragungen (Fig. 4b.)

Gleich darauf (Fig. 4c) glätten die Einfaltungen der Kernwand sich aus; an der Zelloberfläche treten grössere und zum Teil lappige Exkreszenzen auf.

Diese erscheinen auf einem folgenden Stadium (Fig. 4d) an dem einen (in Fig. $4 d$ obern) Pol der noch immer leicht ellipsoidischen Zelle in grösserer Zahl, während sie an der übrigen 
Zelloberfläche mehr und mehr eingezogen werden. Der kugelig gewordene Kern begibt sich in eine exzentrische Lagerung; er entfernt sich von demjenigen Pol, an welchem die zahlreichen Protoplasmafortsätze aufgetreten sind, und verlagert sich an die gegenüberliegende Seite.

Auf dem Stadium der Fig. $4 \mathrm{e}$ hat auch der Zelleib eine kugelige Form. Die Zelloberfläche geht noch immer deutlich erkennbar unter der Basis der Protoplasmafortsätze weg. An Zellen auf dem gleichen Stadium der Veränderung wie Figg. $4 \mathrm{c}-\mathrm{e}$, welche mit Sublimat fixiert und mit Ehrlich-Biondischer Lösung gefärbt sind (Figg. 18, 19), fällt sie sogar durch ihre starke Tinktion auf, welche an der ebenso behandelten unverănderten Zelle (Fig. 17) nicht vorhanden ist.

Danach (Fig. 4f) treten schlankere Fortsătze auf, die offenbar neu ausgesandt werden. Sämtliche Fortsätze lassen nunmehr deutlich eine Zentrierung auf einen neben dem Kern liegenden Punkt erkennen. Die Zelloberfläche unter der Basis der Fortsătze entzieht sich dem Blick.

Das Stadium, welches sich an dasjenige der Fig. $4 \mathrm{f}$ anschliesst (zirka 15 Minuten nach Entnahme des Blutes) zeigt folgendes Bild (Fig. 5-12).

Neben dem Kern liegt ein Cytoplasmaklümpchen, welches diesem oder auch zugleich der Oberfläche des Objektträgers anklebt und nach den übrigen Seiten zahlreiche radiär gestellte fadenförmige Fortsătze aussendet. Die meisten von ihnen sind an ihrem Ende leicht keulenförmig verdickt; andere, welche gewöhnlich länger sind, endigen zugespitzt. Neben den fadenförmigen Fortsätzen sind meistens noch mehr voluminöse vorhanden (Fig. 6-12), welche sich hauptsăchlich nach der Seite hin erstrecken. Besonders diese letzteren zeigen fortwährende Formänderungen; einzelne von ihnen können sich als helle Bläschen abschnüren. Zuweilen sieht man auch ,wie zerrissen aussehende Anhängsel" (Fig. 7 und 10).

An der übrigen Peripherie ist der Kern entweder vollstăndig nackt oder teilweise oder ganz von einem hyalinen, hier und da eingebuchteten Protoplasmasaum umgeben, welcher, ebenso wie die plumpen Fortsătze, lebhaftere Bewegung zeigt.

Fasst man die Konturen des Kerns ins Auge, so erkennt man, dass er seine Gestalt von dem Augenblick an, wo er sich 
abgerundet hat, weiter ununterbrochen ändert. Mit oder nach der Formierung des Cytoplasmaklümpchens treten häufig Knospungsvorgänge an ihm auf; zwei oder auch drei kleine Knospen schnüren sich nacheinander oder auch gleichzeitig von ihm ab. und zwar konstant an der dem Cytoplasmaklümpchen zugekehrten Seite (Fig. 6, 7, 9, 14). Anderemale beobachtet man, dass sich der ganze Kern in eine Anzahl ungleich grosser Stücke fragmentiert (Fig. 10 und 11).

Spindelzellen auf dem zuletzt beschriebenen Stadium der Veranderung sind in den Figg. 21-26 nach Fixierung in Sublimat, in den Figg. 32-37, 39 und 40 nach Behandlung mit Fle m min gschem Gemisch dargestellt. Über die Wirkungsweise der angewandten Reagentien vergl. S. 315. Fig. 20 zeigt einen nicht ganz selten vorkommenden Fall, in welchem sich zwei Cytoplasmaklümpchen an zwei voneinander entfernten Stellen der Kernperipherie entwickelt haben.

Knospungs- und Fragmentierungsvorgänge des Kerns in fixiertem Zustand sind in den Figg. 19, 22-28, 35, 40 und 46 zu sehen.

Neues erfährt man an den fixierten und gefürbten Präparaten besonders bezüglich des Verhaltens des am frischen Objekt unsichtbaren Kernchromatins.

Dieses bildet an der dem Cytoplasmaklümpchen zugekehrten Seite des Kerns regelmüssig eine grössere liompakte Masse, unter der übrigen Kernoberfläche dagegen nach wie vor eine ununterbrochene Schicht von etwa der gleichen Dicke wie am Kern der unveränderten Zelle. Das Kerninnere enthäit im übrigen wenig oder gar kein Chromatin mehr. Man wird daher annehmen dürfen, dass die grössere Chromatinmasse durch Zusammenhäufung der feinen Körnchen entstanden ist, welche im Kern der unveränderten Zelle verstreut sind.

Mit der beschriebenen Anordnung des Chromatins hängt es offenbar zusammen, dass die Kernknospen, welche sich, wie erwähnt, stets von der dem Cytoplasmaklümpchen zugekehrten Seite abschnüren, ganz oder grösstenteils aus Chromatin bestehen. -

In den Spindelzellhaufen verändern sich die einzelnen Zellen ebenso wie die isoliert liegenden; nach den Bildern, welche die in Sublimat fixierten Zellbaufen zeigen (Fig. 27 und 28), 
scheint ihr Protoplasma meistens nicht so vollständig zu verschmelzen, wie es nach dem frischen Objekt den Anschein hat. -

Das zuletzt geschilderte Aussehen können die Spindelzellen im extravasculären Blut stundenlang beibehalten. Dann erst treten Erscheinungen auf, welche auf eingetretenen Tod hindeuten: Kern und Cytoplasma quellen und nehmen ein blasses, verschwommenes Aussehen an; die Cytoplasmafortsätze erleiden hăufig einen körnigen Zerfall.

Die Veränderungen dagegen, welche sich an den Spindelzellen gleich nach dem Austritt des Blutes aus dem Körper abspielen, dürfen meines Erachtens nicht als Degenerations- oder Absterbeerscheinungen aufgefasst werden; ich sehe in ihnen vielmehr Lebensvorgänge, welche der Ausdruck einer bestimmten funktionellen Tätigkeit sind, wenn sie auch den Untergang der Zelle im Gefolge haben. -

Einen abnormen Verlauf scheint die Veränderung der Spindelzelle in dem in Fig. 38 abgebildeten Fall genommen zu haben; ahnliche Bilder habe ich in fixierten Präparaten wiederholt beobachtet. Der Kern ist stark vergrössert und rings von Protoplasma umgeben, welches lappige Exkreszenzen bildet. Man könnte zweifelhaft sein, ob hier wirklich eine veränderte Spindelzelle und nicht vielleicht ein Leukocyt vorliegt; die Diagnose Spindelzelle wird jedoch durch die nach allen Seiten hin abgehende feine Fibrinstrahlung (vergl. S. 340 u. folg.) gesichert.

Zuweilen trifft man $1 / 4-1 / 2$ Stunde nach Herstellung des Präparates vereinzelte Zellen, welche ein Bild wie das in Fig. 13 gezeichnete darbieten. Der Kern ist gequollen und von einem hyalinen Hof (,Halo") umgeben, das Cytoplasmaklümpchen dagegen anscheinend unverändert. Eine ebensolche Zelle nach Fixierung in Flemmingschem Gemisch ist in Fig. 42 dargestellt. Die Erscheinungen, welche sich hier abgespielt haben, deuten offenbar auf vorzeitiges Absterben; sie sind mit den 1901 von Dekhuyzen geschilderten identisch, welche unter dem Einfluss. einer isotonischen, besser einer hypotonischen Kochsalzlösung zustande kommen (vergl. S. 329 und Textfigur b). Von Dekhuyzen werden sie zutreffend als ,agonale“ bezeichnet. $O b$ es aber gerechtfertigt ist, ihnen, wie Dekhuyzen will, funktionelle Bedeutung beizulegen, möchte ich bezweifeln, weil sie im zusatzfreien Blut fast immer nur vereinzelt und relativ spät auftreten. 


\section{Thrombocyten und Gerinnung.}

Über die feineren histologischen Vorgänge bei der Gerinnung existiert eine grosse Literatur; soweit sie sich auf Säugeti erblut bezieht, kann hier nur einiges wenige daraus hervorgehoben werden.

Nach der Lehre von A. Schmidt (77), welche hauptsächlich auf physiologisch-chemischen Erwägungen basiert, wird die Blutgerinnung durch ein Ferment ausgelöst, welches in geringen Mengen im zirkulierenden Blut vorhanden ist, zum grössten Teil aber erst im Augenblick der Gerinnung durch den massenhaften Zerfall von Leukocyten entsteht.

Hayem (78) und Bizzozero (82) kamen dann aber zu dem Resultat, dass nicht die Leukocyten, sondern die Blutplättchen bei der Gerinnung beteiligt sind. Sie beriefen sich dafür unter anderem auf die von M. Schultze (65) gemachte, von Ranvier (73) u. a. bestätigte Beobachtung, dass die Blutplättchen Centren der Fibrinfädenbildung darstellen. Namentlich Bizzozero (82) brachte auf dem Wege des Experiments eine Reihe weiterer gewichtiger Gründe bei, welche für eine Beziehung der Blutplättchen zur Gerinnung sprechen.

Von anderer Seite (Löwit (84) Schimmelbusch (85), Eberth und Schimmelbusch (88)) ist dagegen eine gerinnungserzeugende Wirkung der Blutplättchen bestritten worden. Auch Kopsch (01) findet, dass nach dem morphologischen Verhalten der Blutplättchen bei der Gerinnung die Abhangigkeit der Fibrinbildung von dem „Zerfall" der Blutplättchen sich nicht beweisen lässt. -

In pathologischen Fällen wird die Fibringerinnung nach Hauser (92) und Zenker (95) durch ein aus absterbenden 7ellen sich ausscheidendes Ferment bedingt.

$\mathrm{H}$ auser (92) fand in stark entzündetem Bindegewebe an Präparaten, welche nach der Weigertschen Fibrinfärbungsmethode mit Anilinwasser-Gentianaviolett tingiert waren, ein sehr zierliches, aber fast überall ziemlich engmaschiges Fibrinnetz, in welchem ausserordentlich zahlreiche Knotenpunkte hervortraten, welche bei ganz schwacher Vergrösserung wie rundliche, dunkel gefärbte, in der Peripherie etwas verwaschene, sehr auffallige Flecke erschienen. „Bei stärkerer Vergrösserung 
erkennt man sofort, dass ein jeder dieser blau gefärbten Flecke einer sehr dichten Fibrinablagerung entspricht, welche in ihrer Mitte eine Bindegewebszelle oder ein farbloses Blutkörperchen einschliesst und in der Peripherie sich in nach allen Seiten hin radiür ausstrahlende, mit dem beschriebenen Fibrinnetze in unmittelbarer Verbindung stehende, zarte Fibrinfäden auflöst". Die in den „Gerinnungscentren " eingeschlossenen Zellen zeigen fast ausnahmlos Veränderungen, welche als Erscheinungen des Absterbens zu deuten sind. Analoge Befunde hat $\mathrm{Ha}$ user auch bei der intravaskularen Blutgerinnung gemacht, bei welcher die Fibrinfäden von degenerierten Gefässendothelien sowie von abgestorbenen Leukocyten ausstrahlten.

K. Zenker (95) konnte das ausserordentlich hăufige Vorkommen der. "Gerinnungscentren" bei der interstitiellen und intravasculären Gerinnung bestätigen; bei der letzteren sind es nach ihm namentlich Blutplättchenhaufen, um welche herum Fibrinfaden radiär angeordnet sind.

Gegen die von Hauser und Zenker vertretene Auffassung der „Gerinnungscentren" sind von Arnold (93 und 97,2) Bedenken erhoben worden. Arnold beobachtete eine strahlige Gruppierung von Fibrinfäden auch um Fremdkörper und wurde daher zweifelhaft, „ob solche Bilder als der morphologische Ausdruck eines fibringeneratorischen Vorganges gedeutet werden dürfen und ob sie nicht vielmehr die Vorliebe des Fibrins für gegebene fixe Punkte bei der Ausscheidung anzeigen". Demgegenüber hat $\mathrm{Hauser}(98)$ noch einmal die Gründe erörtert, welche für eine fibringeneratorische Rolle der Gerinnungscentren sprechen. Die Diskussion zwischen Arnold und $\mathrm{Hauser}$ hat sich in zwei Artikeln des Zentralblatts für allgemeine Pathologie und pathologische Anatomie (Bd. 10, 1899) noch weiter fortgesetzt. -

An dieser Stelle muss schliesslich noch eine Arbeit von Laker Erwahnung finden.

Laker, welcher in einer ersten Mitteilung (82) sich in allen wesentlichen Punkten an Bizzozero anschloss, kam später (84) zu dem Resultat, dass der erste Beginn der Blutgerinnung vom Blutplasma ausgeht, ohne dass die Annahme einer Beeintlussung von Seiten der körperlichen Elemente dringend notwendig er- 
scheint. Es gelang ihm nämlich, den Nachweis zu liefern, dass als erste Gerinnungserscheinung auf dem Objektträger eine Fibrinausscheidung in Form einer homogenen, dem Glase dicht anliegenden Membran auftritt, welcher er den Namen "primäre Fibrinmembran" gegeben hat.

Von der Existenz dieser Membran überzeugte Laker sich auf folgende Weise. Er schwemmte einen schnell auf den Objektträger gebrachten Tropfen Blut, obne ihn mit einem Deckgläschen zu bedecken, rasch aber vorsichtig in gleichmässigem Strome mit 1\% iger Osmiumsäure weg, bis mit freiem Auge nichts mehr oder nur eine leise lrübung zu entdecken war. Setzt man nun der Osmiumsäure etwas Methylanilinviolett zu und bedeckt vorsichtig mit einem Deckgläschen, so ist baufig ausser mehr oder weniger gut erhaltenen Blutscheibchen und einzelnen weissen Blutkörperchen auch mit den stärksten Vergrösserungen nichts $z u$ entdecken. Wenn man jedoch vor dem Bedecken mit dem Deckgläschen die Stelle, wo der Bluttropfen adhariert hatte, mit der Spitze einer scharfen Nadel mehrfach durchkratzt, so gelingt es meist ganz gut, die Spuren, welche die Nadelspitze in der primären Fibrinmembran zurückgelassen hat, als Furchen mit leicht aufgeworfenen Rändern, die sich stärker tingieren als die Umgebung, mit stärkeren Vergrösserungen aufzufinden.

Da man einwenden könnte, dass die beschriebene Membran durch Einwirkung der Osmiumsäure zustande kommt, so stellte Laker Kontrollversuche an, bei welchen er als SchwemmHlüssigkeit konzentrierte Magnesiumsulfatlösung benutzte, welche bereits gebildetes Fibrin nicht mehr in Lösung bringt, dagegen als eminent gerinnungshindernd bekannt ist. Bei Anwendung dieser Lösung gelang es ebenfalls, die Fibrinmembran zur Darstellung zu bringen.

Gutschy hat neuerdings (03) die Angaben Lakers über die Bildung einer primären Fibrinmembran mit Hilfe der von Arnold angewandten Methode der Hollundermarkplattchen nachgeprüft und bestätigt gefunden. Auf die Frage; ob im weiteren Verlauf der Blutgerinnung noch andere Faktoren mit in Betracht zu ziehen seien, will Gutschy nicht eingehen. 
Zur Morphologie der Gerinnung des Amphibienblutes finde ich in der Literatur folgende Angaben.

Ranvier (75) nimmt an, dass an Stelle der von roten Blutkörperchen gebildeten Rosetten, welche in einem Präparat von Froschblut einige Zeit nach der Herstellung auftreten (vergl. unten, S. 347), Fibrinmassen gelegen sind; die von den Rosetten ausgehenden Strahlen werden, wie er sagt, von Fibrinfasern gebildet, welche die roten Blutkörperchen festhalten.

Hayem (79) schliesst zunächst auf das Vorhandensein eines in bestimmter Weise angeordneten Fibrinnetzes aus den Erscheinungen, welche sich bei der Gerinnung des Froschbluts an den Spindelzellen und den roten Blutkörperchen in der Umgebung der Spindelzellen abspielen.

Wenn man sich eine Stelle eines frisch hergestellten Präparates' aussucht, wo in demselben Gesichtsfeld mehrere isolierte Spindelzellen in geringem Abstand voneinander liegen, so nimmt man nach $\mathrm{H}$ ayem (nach etwa $2^{1 / 2}$ bis 3 Stunden) wahr, dass die Spindelzellen mehr und mehr zusammenfliessen, wie wenn sie durch die Wirkung von Fäden zueinander hingezogen würden. Diese Fäden sind für gewöhnlich unsichtbar; jedoch sieht man sie bäufig, sobald die Spindelzellen sich einander genähert haben, zu kleinen BündeIn verdichtet in die Erscheinung treten.

Während diese Zusammengruppierung der isolierten Spindelzellen vor sich geht, werden die umliegenden roten Blutkörperchen in gleicher Weise durch unsichtbare Bande angezogen. Einer ebensolchen Anziehung unterliegen sie in der Umgebung der Spindelzellhaufen.

Diese Tatsachen, sagt $\mathrm{Hayem}$, erlauben uns bereits das. Vorhandensein eines Fibrinnetzes anzunehmen, dessen einziehbare Hauptfäden die Spindelzellen unter einander verbinden, während andere von den Knotenpunkten nach allen Seiten hin ausstrahlen, um die roten Blutkörperchen zu umschlingen.

Die Fibrillen dieses Netzes sind so fein, dass es absolut unmöglich ist, sie im frischen Zustande wahrzunehmen ${ }^{1}$ ). Man kann sie jedoch durch leichte Eintrocknung sichtbar machen;

1) Die vereinzelten Fäden, welche man in frischen Präparaten wahrnimmt, sind nach Hayem, wie gesagt, durch Vereinigung mehrerer Fibrillen zu Bündeln entstanden. 
ferner dadurch, dass man durch ein Blutpräparat, welches man eine zeitlang unter einer feuchten Glocke aufbewahrt hat, Jodserum hindurchzieht, durch welches eine Anzahl roter Blut-

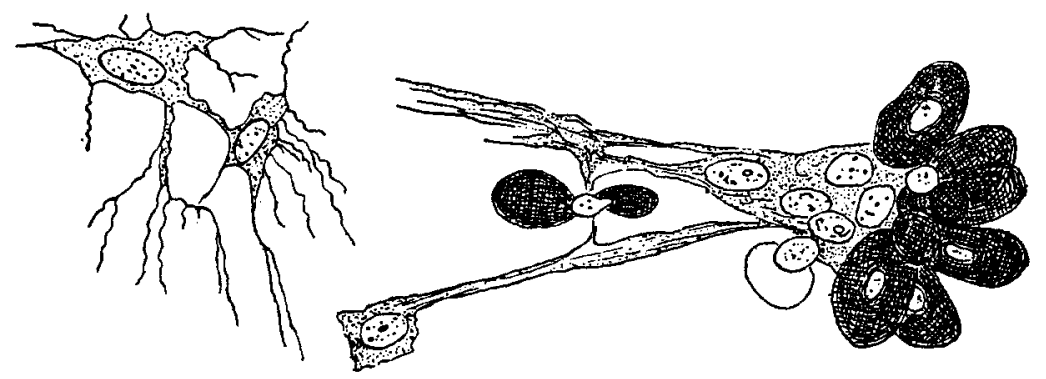

Fig. c.

Geronnenes Froschblut, teilweise eingetrocknet. Nach Haye m, Du sang, S. 148.

körperchen fortgeschwemmt werden, und dieses später durch eine Jodjodkalilösung ersetzt. Bei dem letzteren Verfahren färben sich alle Elemente sofort gelb, und man sieht von den Spindelzellen, welche sich zu eckigen und sternförmigen Körperchen

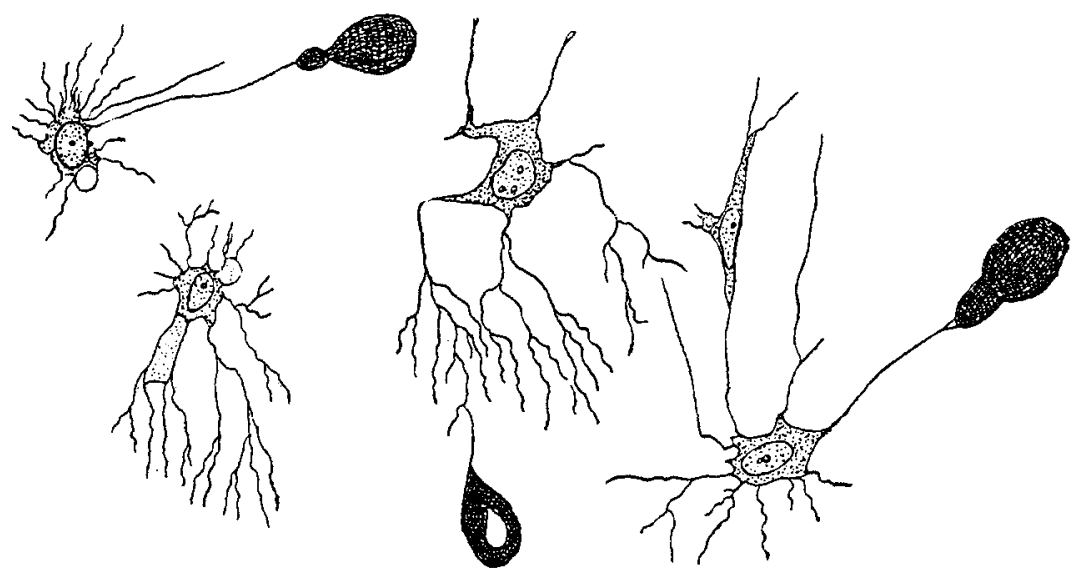

Fig. d.

Spindelzellen aus geronnenem Froschblut, mit Jodserum behandelt.

Nach Hayem, Du sang, S. 149.

umgeformt haben, zahlreiche Fäden ausgehen, welche sich teilen und wieder teilen in Fibrillen von ausserster Zartheit, die nur infolge der durch das Jod erzeugten gelben Färbung kenntlich werden. 
Bizzozero (82, S. 326) will auf die morphologischen Vorgånge bei der Gerinnung des Froschblutes nicht eingehen, da sie bereits ausführlich von $\mathrm{H}$ a y e m beschrieben seien; er nimmt seinerseits eine Beteiligung der Spindelzellen bei diesem Prozess auf Grund von Versuchen an.

Nach Hlava (83, S. 414-415) ist die Blutgerinnung eine „Coagulationsnekrose" der weissen Blutkörperchen, durch welche das Ferment frei wird, welches mit Stoffen des Plasmas das Fibrin hervorbringt. Die Spindelzellen des Froschbluts beteiligen sich an der Blutgerinnung insofern, als sie eine Art weisser Blutkörperchen sind; ihre Beteiligung ist jedoch eine höchst minimale.

Eberth und Schim melbusch (88, S. 72) stellen jegliche Beteiligung der Spindelzellen (ebenso wie der Blutplättchen des Säugetierblutes) bei der Gerinnung in Abrede. Die im Froschblut auftretenden Faserstofffäden setzen sich an alle festen Punkte an, so auch an die verschmolzenen Spindelhäufchen. „Es ist die Faserstoffabscheidung beim Frosch aber nur mit Schwierigkeit $\mathrm{zu}$ erkennen und bietet lange nicht das in die Augen fallende Bild wie beim Warmblüter."

Griesbach $(91$, S. 539) konstatierte bei der Gerinnung des Amphibienblutes an den Leukocyten ebensolche Erscheinungen von Plasmoschise, wie sie von Lö wit und ihm selbst an den Blutkörperchen des Krebses und acephaler Mollusken beobachtet waren.

Arnold hat Abschnürungs- und Ausscheidungsvorgänge, wie er sie zuerst (96) an den roten Blutkörperchen von Säugetieren gesehen bat, in einer späteren Arbeit $(97,1)$ auch an denjenigen des Frosches wahrgenommen. Die von ihm mitgeteilten Beobachtungen rechtfertigen nach seiner Ansicht die Annahme, dass die roten Blutkörperchen des Frosches bei der Gerinnung in hervorragender Weise beteiligt sind, unbeschadet der Rolle, welche dabei die Leukocyten, Spindeln und vielleicht noch andere Gewebszellen spielen.

„Bezüglich der Beteiligung der Spindeln", sagt Arnold. $(97,1$, S. 492-493) ,habe ich zu erwähnen, dass nicht selten von ihren Polen fädige Gebilde abtreten oder hyaline Bänder mit ihnen in Verbindung stehen. Ob aber aus solchen Befunden auf eine Beteiligung der Spindeln an der Gerinnung in dem Sinne geschlossen werden darf, dass die an ibnen haftenden 
Gerinnsel aus einem Zerfall der Zellsubstanz derselben hervorgegangen sind, dünkt mir zweifelhaft. Vorerst hat meines Erachtens die Annahme, dass die Spindeln, wie andere corpusculäre Gebilde auch, nur als Centra für die Gerinnung in physikalischem Sinne gedient haben, dieselbe Berechtigung. Selbstverständlich soll durch diese Erwägung die Möglichkeit nicht in Abrede gestellt werden, dass die Spindeln gerade so gut wie andere Zellen durch Ausscheidungen oder selbst durch Zerfall Material zur Gerinnung liefern; ob ihnen aber in dieser Hinsicht ausschliesslich, wie manche meinen, eine Bedeutung zukommt, muss ich als fraglich bezeichnen."

E. Schwalbe (99, S. 101) konnte die Ansichten Arnolds bezüglich der Beteiligung der roten Blutkörperchen bei der Gerinnung bestätigen.

Ich selbst habe den Vorgang der Fibringerinnung an Salamanderblut studiert, welches ich auf dem Objektträger ausgebreitet, in der feuchten Kammer verschieden lange Zeit (ein paar Minuten bis zu mehreren Stunden) sich selbst überlassen, dann mit Flemmingschem Gemisch fixiert und in der oben mitgeteilten Weise gefärbt hatte.

Wenn man ein derartig hergerichtetes Präparat unter das Vikroskop bringt, gewahrt man eine bei schwacher Vergrösserung homogen aussehende Schicht, welche die Oberfläche des Objektträgers überzieht und die zelligen Elemente des Blutes einschliesst; bei Untersuchung mit starkem System weist sie eine körnige, körnig-netzige oder fädig-netzige Struktur auf. Die roten Blutkörperchen füllen die Höhlen der Schicht, in denen sie liegen, meistens nicht vollstandig aus, sondern es bleibt zwischen ihrer Aussenwand und der Innenwand der Höhlen ein heller Zwischenraum (Fig. 31).

Anfangs war ich der Meinung, dass diese Schicht eine durch das Flemmingsche Gemisch erzeugte Niederschlagsmembran sei. Dann machte ich aber sehr bald eine Beobachtung, welche beweist, dass sie schon vor der Fixierung vorhanden sein muss, und kam zu dem Resultat, dass es sich um die primäre Fibrinmembran Lakers (vergl. oben S. 334) handelt.

Ich fand nämlich, dass Leukocyten, welche in der feuchten Kammer zu kriechen angefangen hatten, sich vielfach in der 
in Rede stehenden Membran Gänge gebahnt hatten ${ }^{1}$ ) (Fig. 31). Andere Male hatten sie nur den Ausgangsplatz als Loch zurückgelassen; sie waren dann offenbar a uf der Fibrinmembran weiter gekrochen.

Ein aus dem Serum gebildeter Niederschlag, wie er ja bei Fixierung der Blutpräparate mit Flemming schen Gemisch notwendig auftreten muss, liegt in Gestalt körniger Massen hier und da, zuweilen reichlich, über der Fibrinmembran (diese Niederschläge sind in den Figuren der Tafel 24 und 25 nicht mitgezeichnet).

Von der oben erwähnten Struktur, welche an der Fibrinmembran mit starkem System sichtbar ist, muss ich es dahin gestellt sein lassen, ob sie präformiert ist oder ein Reagentienprodukt darstellt.

Die vorhin erwähnten Hohlräume, welche die in der Fibrinmembran eingeschlossenen roten Blutkörperchen umgeben, sind möglicherweise durch eine nach der Bildung der Fibrinmembran eintretende Zusammenziehung derselben (eine solche wurde auch von Gutschy 03, S. 35 beobachtet) zustande gekommen.

Sucht man nun in den Präparaten die veränderten Spindelzellen auf, so fällt zunächst auf, dass sie im Gegensatz zu den roten Blutkörperchen innerhalb der primären Fibrinmembran niemals von einem Hohlraum umgeben sind.

Weiter entdeckt man, dass das dem Kern ansitzende Cytoplasmaklümpchen (dessen Fortsätze nach der Fixierung in Flemming schem Gemisch meistens gequollen sind, vergl. oben S. 315) das Zentrum einer nach allen Seiten sich erstreckenden Strahlung bildet; diese ist bereits zwei Minuten nach Entnahme des Bluts (Fig. 32) vorhanden. Die Radien der Strahlung (Fig. 32 u. folg.) sind ausserordentlich fein und anscheinend völlig unverästelt; ihre Länge beträgt etwas mehr als diejenige eines roten Blutkörperchens. Sie sind in einer Ebene ausgebreitet und so zablreich, dass sie einen dichten Schirm bilden. Sie liegen nicht unmittelbar auf der Oberfläche des Glases, sondern auf derjenigen der Fibrinmembran; diese zeigt aber unter dem Strahlenschirm häufig eine geringere Dichte als ausserhalb seines Bereichs; man

1) Der aus aneinander gereihten Löchern bestehende Gang in Fig. 31 erweist sich beim Heben des Tubus. als vollständig geschlossen. 
betrachte die Fig. 35, in welcher rechts oben ein Ausschnitt der Strahlung weggelassen ist.

Entsprechend ihrer oberflächlichen Lage ziehen die Strahlen über den roten Blutkörperchen und Leukocyten, die in der Nachbarschaft der Spindelzellen gelegen sind, hinweg (Fig. 37). Von diesen selbst geht niemals auch nur die Spur einer ähnlichen Strahlung aus wie von den Spindelzellen. Dagegen trifft man zuweilen kleine kernlose Cytoplasmastückchen, welche ebenfalls eine Strahlung um sich entwickelt haben (Fig. 41); von diesen darf aber mit Bestimmtheit angenommen werden, dass sie in der oben S. 318 geschilderten Weise von Spindelzellen abgetrennt worden sind.

Zwischen benachbarten Thrombocyten oder Thrombocytenhaufen bildet sich regelmässig eine Art Spindelfigur aus, wie Fig. 39 zeigt.

Schliesslich sei noch bemerkt, dass die geschilderte StrahIung auch an Sublimatpräparaten, welche mit zu starker EhrlichBiondischer Lösung überfärbt sind, wahrzunehmen ist.

Es fragt sich nun, welcher Natur die Strahlen sind und wie sie entstehen.

Dass es sich um Fäden handelt und nicht etwa nur um den durch das Reagens fixierten Ausdruck von "Strombahnen", dass diese Fäden, wenn sie auch im frischen Präparat nicht sichtbar sind, hier bereits existieren und nicht bloss ein Artefakt darstellen, ergibt sich zweifellos aus den später zu schildernden Deformationen, welche sie an den roten Blutkörperchen in nächster Umgebung der Spindelzellen hervorrufen. Die Strahlen sind ferner jedenfalls keine Fortsetzungen des Cytoplasmas, sondern bestehen höchstwahrscheinlich aus abgeschiedenem Fibrin, wofür auch ihre allmählich eintretende Zusammenziehung (vergl. unten S. 351) spricht; jedoch sind sie mit den ,Fibrinfasern" der Autoren nicht identisch.

Ihr Auftreten muss in ursächlicher Beziehung zu den Spindelzellen stehen und ist meines Erachtens ein bestimmter Beweis dafür, dass die Spindelzellen am Gerinnungsvorgang beteiligt sind. Von dem Cytoplasma der Spindelzellen wird offenbar ein Stoff abgeschieden, welcher mit Bestandteilen der umgebenden Flüssigkeit eine unlösliche Verbindung (Fibrin) bildet. Dass es unter diesen Bedin- 
gungen zur Entstehung von Strahlungen kommen kann, wird durch Versuche bewiesen, welche Alf. F ischer 1899 in seinem Buche „Fixierung, Färbung und Bau des Protoplasmas" beschrieben hat.

Alf. Fischer unterscheidet zwei verschiedene Arten von. Fällungsstrahlungen, welche in Eiweisslösungen zustande kommen können : 1. eine „Fremdstrahlung“", welche in einer übersattigten Lösung um einen heterogenen Körper entsteht, der genau so wirkt wie ein Staubteilchen, das eine übersättigte Salzlösung zur Krystallisation treibt; 2. eine „Selbststrahlung", welche sich um die Diffusionszone des Fallungsmittels bildet. Auf die ,Selbststrahlung:", welche für den uns vorliegenden Fall allein in betracht kommt, beziehen sich zwei von A lf. Fis cher angestellte Versuche. „Auf einem Objektträger wird ein quadratischer Raum durch einen Vaselinwall umgrenzt und dahinein eine kurze, beiderseits offene Kapillare so eingedrückt, dass die eine Mündung kurz in den Innenraum, die andere nach aussen vorragt. Nachdem die Durchtrittsstelle der Kapillare durch das Vaselin noch hoch damit bedeckt worden ist, wird Albumoselösung in den Raum gebracht und ein Deckglas sanft aufgedrückt. Die Albumoselösung muss den Raum innerhalb des Vaselins ganz und ohne Luftblasen erfüllen und darf aus der Kapillare nach aussen nicht hervorströmen. Man hat nunmehr nach kurzer Zeit. eine ganz ruhige Lösung in der kleinen Kammer, die mit schwacher Vergrösserung so eingestellt wird, dass der in die Albumoselösung ragende Mund der Kapillare gut zu sehen ist. Nunmehr setzt man an den freien Kapillarrand einen Tropfen eines Fixierungsmittels, z. B. $0,2 \%$ ige Pikrinsäure, $0,5 \%$ iges $\mathrm{HgCl}_{2}, 1 \%$ iges $\mathrm{PtCl}_{4}, 1 \%$ ige Osmiumsäure, Fle m mingsche Lösung. Mehr wurde nicht geprüft. Von dem Kapillarmunde diffundiert die fixierende Lösung kugelschalig in die Albumoselösung und erzeugt nun hier eine zunächst sehr sanfte Trübung, die bald stärker wird. Schliesslich entsteht ein mehr oder weniger deutliches, nach der Kapillare konvergierendes Strahlensystem ...."

Ein weiterer Versuch ist folgender:

„In den vaselinumgrenzten Raum mit der Albumoselösung tauche man ein sehr kleines Kryställchen von Sublimat unter und lege schnell ein Deckglas auf, wieder so, dass Luftblasen und Strömungen feblen. Um das allmählich sich lösende Kryställchen bildet sich ein Diffusions- und Fallungsgürtel, der nach wenigen 
Minuten schon strahlig wird. Später, nach 10 bis 20 Minuten bildet der ungelöste Rest des Sublimates das Zentrum einer Strahlenfigur ....."

Bei beiden Versuchen besteht das Prinzip darin, dass in eine Lösung ein Zentrum eingetaucht wird, von dem aus das Fällungsmittel diffundiert. Hier bedarf es keines besonderen Strahlenerweckers wie bei den Fremdstrahlungen, sondern die ersten Fälungen, die entstehen, dienen selbst dazu.

Auch die Strahlung, welche um die Spindelzelle zustande kommt, ist meines Erachtens als Selbststrahlung im Sinne von Alf. Fi is ch er aufzufassen; das Cytoplasmaklümpchen der Spindelzelle wirkt in dem extravasierten Blut nach Art eines in Albumoselösung gelegten Sublimatkryställchens. Zuerst tritt die primäre Fibrinmembran auf. Dann diffundiert aus dem Cytoplasma der Spindelzellen eine Substanz ( Fibrinferment"?), welche die feine Fibrinstrahlung mit einer anderen Substanz bildet, die sich an der Oberfläche der Fibrinmembran findet. Letztere Substanz wird ausserhalb des Diffusionsbereiches der Spindelzellen wahrscheinlichs ganz oder grösstenteils zur weiteren Verdichtung der Fibrinmembran verwendet; dadurch, dass sie innerhalb dieses Bereichs von dem diffundierenden Stoff beschlagnahmt wird, erklärt sich wohl die oben erwähnte Tatsache, dass die Fibrinmembran unter dem Strahlenschirm vielfach eine mehr lockere Beschaffenheit zeigt. ${ }^{1}$ )

Dass es zwischen benachbarten Strablenzentren zur Ausbildung einer Spindelfigur kommt (Fig. 39), steht durchaus im Einklang mit Beobachtungen, die Alf. Fischer (l. c. S. 221) beschrieben hat. Durch eine eintretende Verkürzung der Spindelfasern kann möglicherweise eine Annäherung zweier Nachbarzellen, wie sie von Hayem beobachtet wurde, bewirkt werden.

1) Nachdem ich im obigen wiederum die von Fischer experimentell erzeugten Strahlungen herangezogen habe, um histologische Strahlungen abzuleiten (zum ersten Mal zur Erklärung der circumnucleären Strahlungen, welche in den roten Blutkörperchen von Amphibien nach Reagentienzusatz auftreten können, Anat. Anz. Bd. 26, 1905, S. 537), möchte ich daranf hinweisen, dass ich F is cher nicht beistimmen kann, wenn er auch die Spindelfasern und Polstrahlen der achromatischen Figur der Mitose auf solche Ursachen zurückführen will, welche die künstliche Strahlung hervorrufen (vergl. Fr. Meves: Bericht über ,Zellteilung“, Ergebnisse d.Anat. u. Entwicklungsgesch. Bd. 8, 1898, Wiesbaden 1899, S. 534-う35).

Archiv f. mikrosk. Anat. Bd. 68. 


$$
344 \text { Friedrich Meves: }
$$

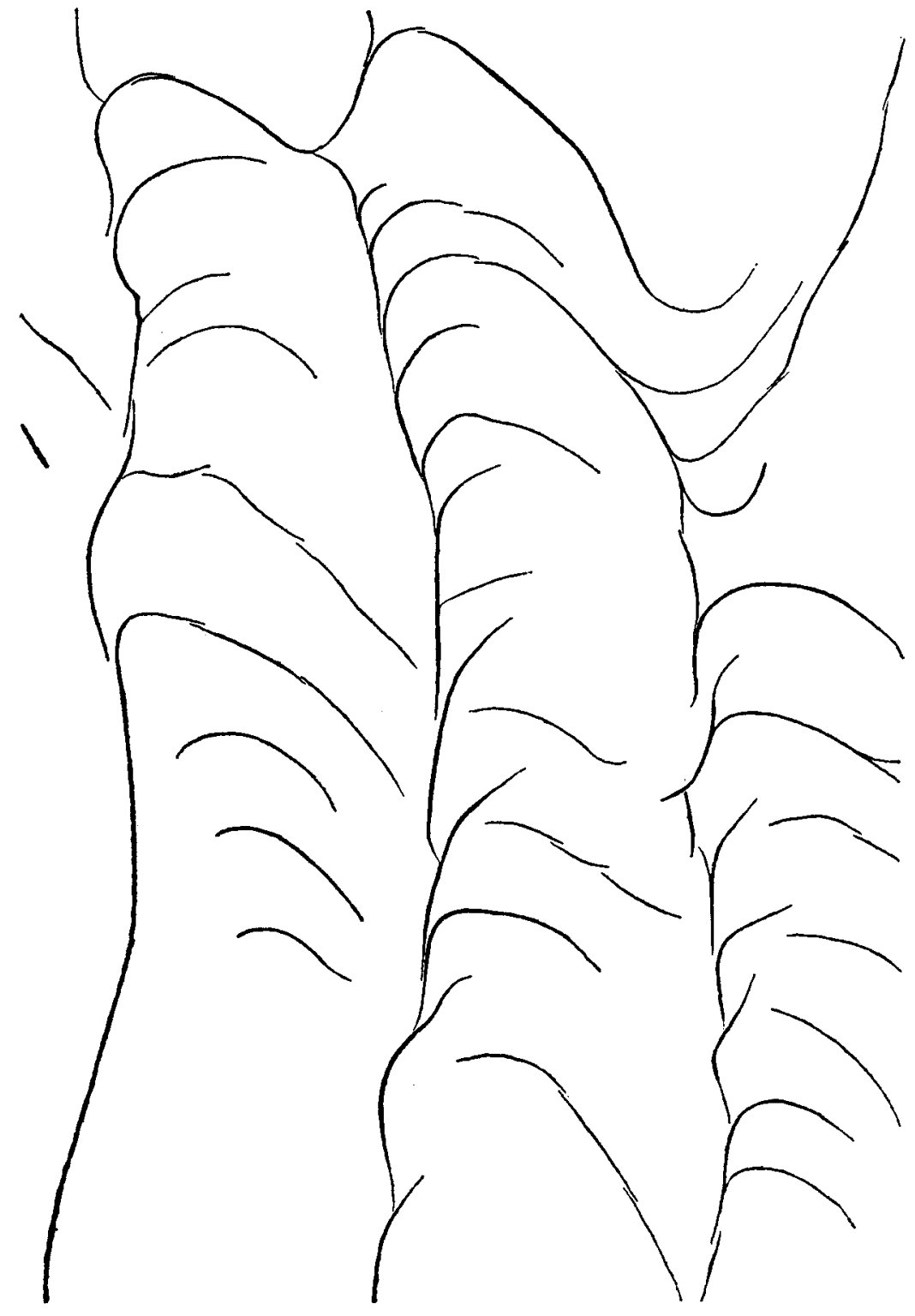

Fig. e.

Fibrinfasern aus einem mit $\mathrm{Fl}$ e m ming schem Gemisch fixierten Präparat von Salamanderblut.

Ausser den eben beschriebenen feinen Fibrinfäden, die radiär um das Cytoplasmaklümpchen der Spindelzelle angeordnet 
sind, kommen auch die gewöhnlichen „Fibrinfasern" zur Beobachtung. Allerdings gibt es immer zahlreiche Stellen des Präparates, wo sie gänzlich fehlen, und ganze Präparate, in denen sie nur sehr spärlich zu finden sind. Sie präsentieren sich als dickere Fäden von verschiedener Lănge, welche meistens nicht gerade, sondern mehr oder minder stark bogenförmig verlaufen.

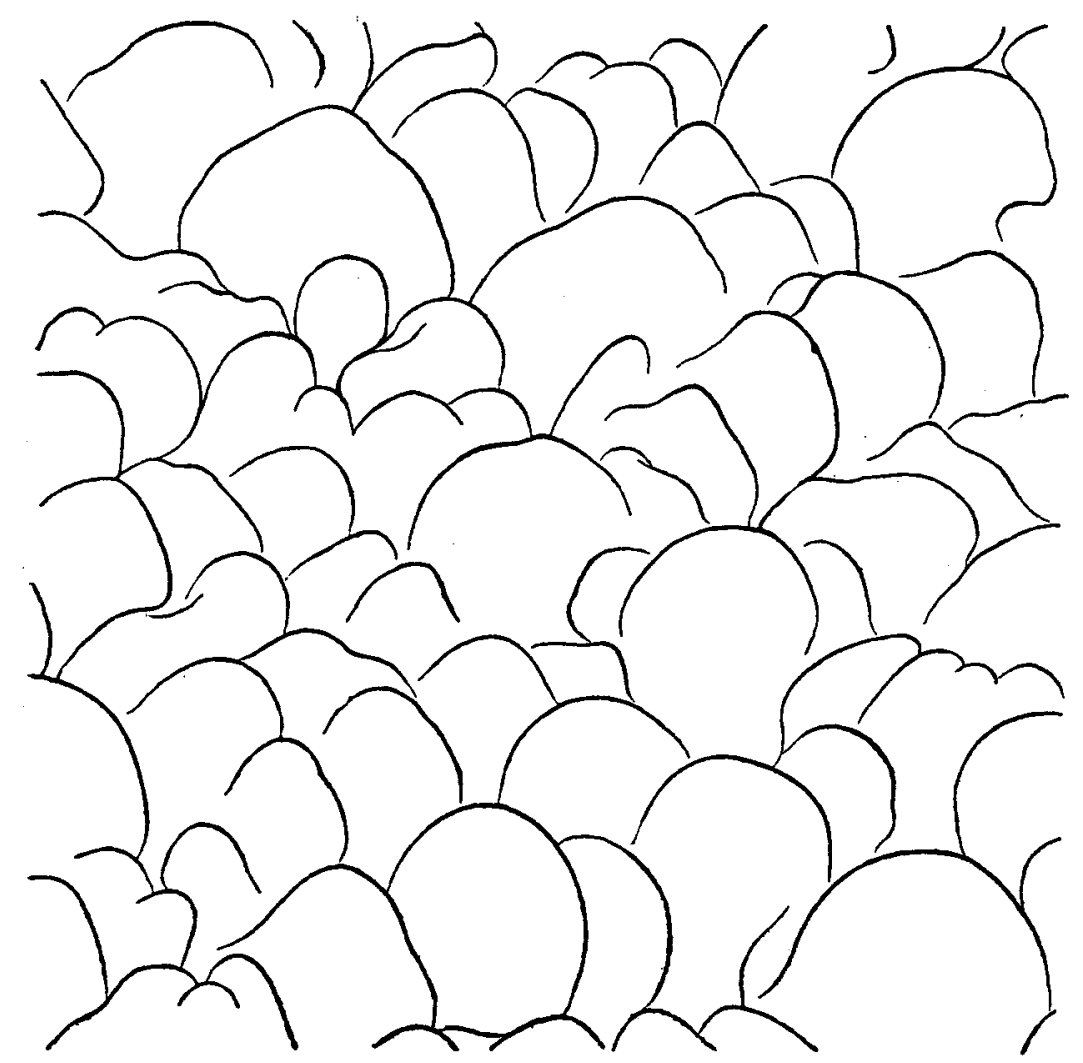

Fig. f.

Netz von Fibrinfasern, aus einem mit Flemming schem Gemisch fixierten Präparat von Salamanderblut.

Gewöhnlich spitzen sie sich nach den Enden hin allmăhlich zu. Sie sind stets über der primären Fibrinmembran gelegen. Sie laufen entweder isoliert oder legen sich (entweder mit ihren Enden oder in der in Fig. e abgebildeten Weise) zu Fäden aneinander; dadurch, dass die Zwischenrăume zwischen benach- 
barten Fäden von anderen überquert werden oder durch eine Lagerung der einzelnen Fasern wie in Fig. $f$ wird der Eindruck eines Netzes hervorgebracht.

Wo diese Fasern zusammen mit Spindelzellen an ein und derselben Stelle des Präparates vorkommen, sind sie noch ü b e r der von dem Cytoplasmaklümpchen der Spindelzelle ausgehenden feinen Fibrinstrahlung gelegen. Häufig sind sie radiär zum Cytoplasmaklümpchen orientiert und scheinen an ihm mit ihrem einen Ende zu inserieren (Fig. $35,36,39,40$ ). In diesem Fall könnte man mitunter zu dem Glauben kommen, sie seien durch Verschmelzung feiner Fäden entstanden, besonders, wenn ihr Verlauf ein gerader ist und man sich noch nicht davon überzeugt hat, dass sie in einer höheren Ebene liegen. Andere Male aber gehen sie über das Cytoplasmaklümpchen hinweg oder ziehen an ihm vorbei, indem sie quer zur Richtung der feinen Strahlen verlaufen.

Dass die Fibrinfasern Faltungen der Fibrinmembran seien, wie Laker meint, ist gänzlich ausgeschlossen (vergl. auch Schimmelbusch 85 S. 236 und Eberth und Schimmelbusch 88 S. 41).

Das Fibrin würde demnach in einer auf dem Objektträger ausgestrichenen Schicht von Salamanderblut in drei Formationen auftreten, welche sich in verschiedener Höhe lagern: als primäre Fibrinmembran an der Oberfläche des Glases; als feine Fibrinstrahlung an der Oberfläche der primären ribrinmembran; als dickere Fibrinfasern ebenfalls an der Oberfläche der primären Fibrinmembran und noch oberhalb der feinen Fibrinstrablung, wo sie mit dieser zusammen vorkommen. -

Ha y e $m$ ist bisher der einzige gewesen, welcher auf Grund morphologischer Beobachtungen behauptet hat, dass die Spindelzèllen bei der Gerinnung eine Rolle spielen und zwar hat er diese Behauptung mit der Existenz eines von den Spindelzellen ausgehenden Fibrinnetzes begründet. Dabei hat er offenbar, wie aus seiner oben referierten Beschreibung und aus seinen Abbildungen hervorgeht, ein Netz dickerer Fibrinfasern, von der Art wie es meine Fig. 40 zeigt, im Auge gehabt; die von mir beschriebene feine Fibrinstrahlung, auf welche ic $\mathrm{h}$ mich mit der gleichèn Behauptung stütze, hat er dagegen nicht zu sehen bekommen. Die dickeren Fibrinfäden zeigen, nun aber, wie ich 
oben geschildert habe, eine sehr wechselnde Anordnung; ein radiäres Ausstrahlen wie in Fig. 40 ist keineswegs die Regel. Ein Zusammenhang zwischen den Spindelzellen und der Bildung dieser dickeren Fibrinfasern lässt sich daber meines Erachtens nicht beweisen.

Noch weniger kann auf Grund der morphologischen Daten behauptet werden, dass die Spindelzellen bei der Entstehung der primären Fibrinmembran beteiligt sind.

Dafür, dass die Leukocyten und roten Blutkörperchen in irgend einer ursächlichen Beziehung zur Gerinnung stehen, habe ich in meinen Präparaten keine Anbaltspunkte gefunden.

\section{Thrombocyten und rote Blutkörperchen.}

Ranvier hat 1875 (S. 215) beschrieben, dass die roten Blutkörperchen in einem Präparat von Froschblut nach 15-20 Stunden eine bestimmte regelmässige Anordnung zeigen: An gewissen Punkten sind sie zu einer kleinen rundlichen Masse angeordnet, von eleganter Form ähnlich einer Rosette, von deren Rändern Strahlen ausgehen, welche sich mit ähnlichen Strahlen einer benachbarten Rosette verbinden.

Die roten Blutkörperchen, welche die Rosette bilden, sind den frei im Präparate schwimmenden unähnlich. Sie sind kugelig, stärker gefärbt, stärker lichtbrechend und kleiner als die letzteren. Bei 150 facher Vergrösserung hat man Mühe, sie als rote Blutkörperchen za erkennen. Man würde sie einfach für gefärbte Körner halten. Untersucht man aber bei stärkerer Vergrösserung, namentlich aber, wenn man die Blutkörperchen getrennt hatte, indem man die Masse durch Druck in verschiedener Richtung auf das Deckglas in Bewegung setzte, so sieht man leicht, dass sie die Form einer Birne angenommen haben. Sind die Gruppen noch nicht getrennt, so ist das dicke Ende des birnförmig gewordenen Blutkörperchen der Seite des Beobachters zugekehrt, während das dünne Ende in ein feines Netz von Fibrin eingeschlossen ist. Ranvier stellt sich vor, dass diese Formänderungen der roten Blutkörperchen auf folgende Weise entstehen. Das Fibrin hat diese Blutkörperchen bei seiner Bildung umgeben und, als es sich zusammenzog, schnürte es sie ein, sodass ein Teil ihrer Masse nach aussen gedrängt wurde, der dann ganz natürlich eine Kugelgestalt bekam, wie sie eine mit Wasser halbgefüllte Kautschukblase annimmt, wenn man sie mit der Hand umspannt, sodass die Flüssigkeit in den Teil der Blase gedrängt wird, der sich nicht in der Hand befindet.

Zwei Jahre später hat $\mathrm{Fuchs}(77, \mathrm{~S} .91)$ angegeben, dass das Zentrum der rosettenförmigen Gruppen, zu welchen sich die roten Blutkörperchen anordnen, von einer ungefärbten körnigen 
Protoplasmamasse eingenommen wird (er fügt in Klammern ,weisses Blutkörperchen" hinzu). An diese Protoplasmamasse setzen sich Kugeln, welche sich von den roten Blutkörperchen abgeschnürt haben, mit zugespitzten Enden an; diese Kugeln zeigen häufig eine Anzahl gegen die Spitze konvergierender Falten, wie ein zugeschnürter Tabaksbeutel.

$\mathrm{H}$ a y e m (79) ist der erste, welcher erkannt hat, dass das Zentrum der Rosetten von Spindeln oder Spindelhaufen gebildet wird.

Wenn man sich ein Präparat von Froschblut herstellt, beobachtet man, solange das Blut unter dem Deckglas noch in Bewegung ist, dass die Spindelzellen $\mathrm{zu}$ Haufen verkleben, welche am Glase festhaften und Hindernisse bilden, um welche die roten Blutörperchen sich anhäufen. An diesen letzteren hat Hayem in der Umgebung eines Spindelhaufens im Blut von Rana temporaria folgende Veränderungen vor sich gehen sehen.

Ein paar Minuten nach Anfertigung des Präparates erscheinen einzelne rote Blutkörperchen durch Fortsătze, welche von den Spindelzellen ausgehen, festgehalten $\mathrm{zu}$ werden; mehrere sind leicht deformiert, wie wenn sie durch unsichtbare Fäden umflochten wären. Zehn Minuten später haben verschiedene entfernter liegende Blutkörperchen sich dem Spindelhaufen, wie durch Fortsätze angezogen, genähert; andere dagegen haben sich entfernt. Nach einer weiteren Viertelstunde findet man einige Blutkörperchen fest an den Rand des Spindelhaufens angelagert; sie sind auf dem Punkt sich zu fragmentieren, was sie zum Teil ein paar Minuten später wirklich tun. Die Zahl der roten Blutkörperchen, welche gegen den Spindelhaufen angezogen werden, wächst in der Folge immer mehr. Nach Ablauf von ungefähr einer Stunde ist ihre Konfluenz am stärksten, während der Spindelhaufen stark eingeengt ist.

H a y e m sagt: „On pourrait exprimer assez bien l'ensemble des phénomènes qui se passent du côté des globules rouges en disant que ces éléments ont été enlacés par une sorte de filet rayonnant qui, parti de l'amas d'hématoblastes, s'est replié sur lui-même en enserrant dans ses mailles invisibles tous les globules rouges compris dans son territoire." Das Fibrinnetz, welches $\mathrm{Haye} \mathrm{m}$ hierbei im Auge hat, ist wiederum das gröbere meiner Fig. 40. 
Neben den Fibrinfasern spielen aber nach Hayem auch Protoplasmafäden oder -fortsätze (,,appendices"), welche von den Spindelzellen ausgehen, bei der Heranziehung der roten Blutkörperchen eine Rolle. Diese Fortsătze gehen zuweilen sehr weit auf die Suche, Hayem möchte fast sagen auf den Fang roter Blutkörperchen aus. Sie enden mit einer Art Knopf oder auch sie ähneln einem kleinen Bündel und erzeugen an der Oberfläche der benachbarten roten Blutkörperchen charakteristische blasse, rundliche oder streifenförmige Flecke. Je nach der Art und Weise, wie sie an der Oberfläche der Blutkörperchen und um sie herum plaziert sind, rufen sie sehr verschiedene Deformationen hervor. „Fixés à une extrémité ils transforment le globule rouge en une sorte de poire, enroulés transversalement autour de son disque ils en font un bissac ou un corpuscule festonné, disposés dans le sens du grand axe, ils lui donnent la forme d'un éventail plus ou moins fermé; enfin ils peuvent en tirant sur ses deux extrémités le replier sur lui-même à la façon d'une bourse. Puis lorsque la rétraction des filaments est extrême, un grand nombre d'éléments se fragmentent et chacun des fragments prend en général la forme d'un corps globuleux."

Eberth und Schimmelbusch (88, S. 72) tun der strahlenförmigen Gruppierung der roten Blutkörperchen um die Spindelhaufen als einer eigentümlichen Erscheinung Erwähnung, aber nur, ,um die Idee von der Hand zu weisen, als liege hier in diesem ziemlich auffallenden Phänomen eine rătselhafte Fahigkeit der Spindeln vor." Dieselbe Gruppierung roter Blutkörperchen soll nach Eberth und Schimmelbusch im Froschblut um alle Hervorragungen, z. B. auch um zufällige Verunreinigungen des Deckglases, eintreten.

Nach Neumann (96, S. 245) bestehen die Deformationen der roten Blutkörperchen, welche sich im Umkreis der Spindelhaufen gruppieren, teils in zirkulären Einschnürungen derselben mit Faltenbildungen der Zellmembran, wobei die beiden Teile bisweilen vollständig ihren Zusammenhang zu verlieren scheinen. teils in einer Art Korrosion; es zeigen sich kleinere oder grössere, von konkaven Bogenlinien begrenzte Ausschnitte (Defekte) am Rande der Körperchen. Neumann konnte jedoch feststellen, dass diese anscheinend verstümmelten, durchgeschnürten oder defekten roten Blutzellen, wenn sie sich aus der Umgebung der 
Kernhaufen loslösten, wiẹder ihre normale Gestalt und Grösse annahmen. Über die Art und Weise, wie er sich das Zustandekommen dieser Deformationen denkt, macht $\mathrm{Neumann}$ keine Angaben.

Zieler (03) beschäftigte sich auf Veranlassung von Roux mit der Frage nach der Entstehungsursache der radiären Anordnungen roter Blutkörperchen um die Spindelzellenhaufen herum, um zu prüfen, ob hier eine dem von Roux entdeckten Cytotropismus der Furchungszellen vergleichbare Wirkungsweise vorliege. Er kam zu dem Resultat, dass dies nicht der Fall sei. Bei der Entstehung der Sternanordnung spielen nach ihm einmal mechanische Verhältnisse eine Rolle. Dies geht hervor aus der schon von Hayem gemachten Beobachtung feinster von den Sternzentren ausgehender und an rote Blutkörperchen sich anhaftender kontraktiler Fortsätze, die nach $\mathrm{Zieler}$ oft das vierbis sechs- und mehrfache der Länge einer Spindel erreichen sollen.

Als Hauptursache aber kommen wohl chemisch vermittelte zentripetale Strömungen in Frage.

Man kann durch direkte Beobachtung feststellen, dass an den Spindeln und Spindelhaufen deutlich verfolgbare Prozesse vol sich gehen, bestehend in der dauernden Abtrennung kleinster Protoplasmatröpfchen mit folgender Auflösung im Plasma und in sonstigen Protoplasma- und Kernveränderungen; vielleicht werden auch durch moleculäre Umsetzungen chemische Produkte gebildet, die sich ebenfalls in der umgebenden Flüssigkeit auflösen. Durch die Auflösung dieser Zerfallsprodukte entsteht vielleicht eine ganz minimale Strömung von den Sternzentren weg, bei der es sich aber wohl um einen Ausgleich in dem Konzentrationsgrad der verschiedenen Schichten durch allmähliche Diffusion handelt. Da aber durch die gleichzeitig stattfindende Quellung des Protoplasmas und später auch der Kerne der Spindeln, möglicherweise auch zur Lösung der Zerfallsprodukte, Flüssigkeit in reichlicherem Maße gebraucht wird, also von der Peripherie zum Zentrum strömt, so scheint Zi eler der Gedanke naheliegend, dass durch diese Strömung die roten Blutkörperchen mit herangeführt werden. Eine radiäre Einstellung muss bei vorhandener Strömung immer zustande kommen. 
Meinerseits möchte ich im Hinblick auf die gallertartige Beschaffenheit des geronnenen Blutes zunächst behaupten, dass Strömungen, wie sie $\mathrm{Z}$ i e l e r annimmt, hier überhaupt nicht entstehen, jedenfalls aber für das Zustandekommen der Sternanordnung nicht von Bedeutung sein können.

Sodann muss ich gegenüber Hayem und $\mathrm{Zieler}$ in Abrede nehmen, dass die Protoplasmafortsätze der Spindelzellen bei letzterem Vorgang eine Rolle spielen.

Wenn $\mathrm{Zieler}$ angibt, dass diese Fortsätze das vier- bis sechs- und mehrfache einer Spindel erreichen können, so liegt hier offenbar eine Verwechslung mit Fibrinfasern vor. Auch Hayem scheint mir nicht immer klar zwischen Protoplasmafortsätzen und Fibrinfasern unterschieden $\mathrm{zu}$ haben.

Ferner aber habe ich von den schlankeren Protoplasmafortsätzen überhaupt niemals konstatieren können, dass sie irgendeinen Einfluss auf die roten Blutkörperchen ausüben.

Die plumperen dagegen wirken nicht nur nicht anziehend, sondern drängen im Gegenteil gegen die roten Blutkörperchen an und drücken sich mit ihren abgerundeten Enden in die weiche Substanz derselben ein (Fig. 14, 43, 44). Auf diese Weise entstehen an der den Spindelzellen benachbarten Seite der roten Blutkörperchen die von $\mathrm{Neumann}$ erwähnten kleineren oder grösseren, von konkaven Bogenlinien begrenzten Ausschnitte, welche den Eindruck erwecken, als wenn die roten Blutkörperchen korrodiert wären.

Auch ein von den Spindelzellen ausgehendes Netz gröberer Fibrinfasern wie dasjenige meiner Fig. 40, welchem Hayem Bedeutung für die Entstehung der Sternanordnung zuschreibt, kann hierfür nicht in Betracht kommen, da es sich nur verhältnismässig selten findet, die Sternanordnung aber sich um jede Spindelzelle herum ausbildet, in deren Umkreis rote Blutkörperchen liegen.

Meines Erachtens sind die sämtlichen Erscheinungen, welche sich an den roten Blutkörperchen abspielen, mit Ausnahme der bereits besprochenen, auf eine allmähliche Kontraktion der feinen Fibrinstrablen zurückzuführen, welche von dem Cytoplasmaklümpchen der Spindelzellen in radiärer Richtung über die roten Blutkörperchen wegziehen. Dadurch werden die mannigfachen, schon von $\mathrm{Hayem}$ beschriebenen 
Formänderungen der roten Blutkörperchen bedingt, die Faltenbildungen an ihrer Oberflache, welche stets in radiärer Richtung verlaufen, die Zerschnürungen in zwei oder mehr Fragmente, welche zuweilen jeden Zusammenhang $\mathrm{zu}$ verlieren scheinen (Fig. 15, 45, 46).

Nicht selten sieht man die unter Druck geratene Substanz der roten Blutkörperchen hernienartige Aussackungen bilden, welche durch Lücken des Strahlenschirms austreten und oberhalb desselben Kugelform annehmen.

Diejenigen roten Blutkörperchen, in welche sich an der einen Seite Protoplasmafortsätze der Spindelzellen eingelagert haben, zeigen an der entgegengesetzten häufig zablreiche Einkerbungen, auf welche Furchen der Oberfläche zulaufen (Fig. 14, 43, 46). Letztere Erscheinung kommt wahrscheinlich dadurch zustande, dàss die Substanz der roten Blutkörperchen durch die Protoplasmafortsătze vom Sternzentrum weggestaut und gegen die Fibrinfảden, welche in sie einschneiden, angedrängt wird.

\section{Schluss.}

In der vorliegenden Abhandlung ist die Rolle, welche die Spindelzellen bei der Thrombenbildung spielen, unberührt. gelassen.

Bizzozero (82), Hayem (82), Eberth und Schimmelbusch (88) haben gezeigt, dass der weisse Thrombus, dessen Entstehung aus Leukocyten $\mathrm{Zahn}$ (7t) nachgewiesen zu haben glaubte, sich aus Spindelzellen zusammensetzt. Nach B i z z o z e ro. erleiden die den Thrombus bildenden Spindelzellen sekundăre Alterationen. „Ihre Form andert sich, die Kerne werden minder deutlich und die Protoplasmen verschmelzen zu einer einzigen Masse von körnigem Aussehen." Von Eberth und Schimmelbusch werden diese Veränderungen als "viscöse Metamorphose" bezeichnet. $\mathrm{Ob}$ sie identisch mit denjenigen sind, welche im extravasculären Blut auf dem Objektträger vor sich gehen, vermag ich aus der Schilderung der Autoren nicht zu ersehen. Jedenfalls aber möchte ich glauben, dass die sich verändernden Spindelzellen des Thrombus ebenso wie diejenigen des auf den Objektträger gebrachten Blutes eine Substanz abgeben, welche mit Bestandteilen des Blutplasmas Fibrin erzeugt. Demnach würde die Konglutination der Spindelzellen bei der Thrombose 
sich stets mit einer von den Spindelzellen ausgehenden Fibrinbildung kombinieren.

Mit der Aufgabe, welche die Spindelzellen bei der Thrombose und Gerinnung zu erfüllen haben, braucht ihre Bedeutung für den Organismus nicht notwendig erschöpft $z u$ sein. Sie könnten auch unter normalen Verhältnissen irgendwelche Funktionen ausüben. Jedoch ist darüber bisher nichts sicheres festgestellt.

Nach einer Reihe von Autoren (Vulpian, Hayem, Marquis, Neumann, Heinz) sollen die Spindelzellen sich in rote Blutkörperchen umwandeln. Auch Knoll will diese Möglichkeit ,nicht geradezu von der Hand weisen." Die Annahme eines genetischen Zusammenhangs zwischen beiden Zellarten wird hauptsächlich damit begründet, dass im zirkulierenden Blut Zellen vorkommen, welche als intermediäre Formen zwischen Spindelzellen und Erythrocyten aufgefasst werden: hămoglobinhaltige Zellen, welche an einem oder auch an beiden Polen zugespitzt sind; Abbildungen davon haben Hayem (79, Pl. 1, Fig. $5 \mathrm{~d}$ und P1. 5, Fig. 3-5), Knoll (96, Taf. I, Fig. 13-18 und Taf. III, Fig. 23), Heinz (01, Taf. V, Fig. 8) gegeben.

Meines Erachtens handelt es sich aber bei diesen angeblichen Übergangsformen nicht um solche, sondern um rote Blutkörperchen, deren Randreifen eine Läsion erfahren bat ${ }^{1}$ )

\section{Verzeichnis der zitierten Literatur.}

Arnold, J. (93): Über die Geschicke der Lencocyten bei der Fremdkörperembolie. Virchows Archiv, Bd. 133.

Derselbe (96): Zur Morphologie und Biologie der roten Blutkörper. Virchows Arch., Band 14ũ.

Derselbe $(97,1)$ : Die corpusculären Gebilde des Froschblutes und ihr Verhalten bei der Gerinnung. Virchows Arch., Bd. 148.

Derselbe (97,2): Zur Morphologie der extravasculären Gerinnung. Virchows Archiv, Bd. 150 .

Derselbe (99): Über die sogenannten Gerinnungscentren. Centralbl. für allg. Path. und path. Anat., Bd. 10.

1) Vergl. Friedr. Meves: Über das Auftreten von Deformationen des Randreifens bei den roten Blutkörperchen des Salamanders. Anat. Anz. Bd. 25, 1904, S. $471-472$. 
Bizz ozer o, J. (82): Über einen neuen Formbestandteil des Blutes und dessen Rolle bei der Thrombose und der Blutgerinnung. Virchows Archiv, Bd.90.

Deetjen, H. (00): Untersuchungen über die Blutplättchen. Habilitationsschrift, Kiel und Virchows Arch., Bd. 164, 1901.

Dekhuyzen, M. C. (92): Über das Blut der Amphibien. Verh. der anat. Ges. Wien.

Derselbe (01): Über die Thrombocyten (Blutplättchen). Anat. Anz., Bd. 19.

E berth, C. J. (87): Zur Kenntnis der Blutplättchen bei den niederen Wirbeltieren. Festschrift, A. v. Kölliker zur Feier seines 70. Geburtstages gewidmet, Leipzig.

Eberth, C. J. a. C. S chimmeIbusch (88): Die Thrombose nach Versuchen und Leichenbefunden. Stuttgart.

Eisen, G. (97): Plasmocytes; the Survival of the Centrosomes and Archoplasm of the Nucleated Erythrocytes, as Free and Independent Elements in the Blood of Batrachoseps attenuatus Esch. Proceedings of the California Academy of Science. 3 Ser. Zoology Vol. I.

Fuchs, E. (77): Beitrag zur Kenntnis des Froschblutes und der Froschlymphe. Virchows Arch., Bd. 71.

Gigli o-Tos, E. (97): I Plasmociti di Eisen. Anat. Anz. Bd. 14.

Derselbe (98): I Trombociti degli Ittiopsidi e dei Sauropsidi. Memorie della Reale Accademia delle Scienze di Torino, ser. 2, t. 48.

Golubew, A. (68): Über die Erscheinungen, welche elektrische Schläge an den sogenannten farblosen Formbestandteilen des Blutes hervorbringen. Sitzgsber. d. Kais. Akademie d. Wiss., math.-naturw. Cl., Bd. 57, Abt. 2.

Griesbach, H. (91): Beiträge zur Kenntnis des Blates. Pflügers Arch. f. d. ges. Physiologie. Bd. õo.

Derselbe (92): Zur Frage nach der Blutgerinnung. Centralblatt f. d. med. Wissensch. 1892.

Gutschy, L. (03): Zur Morphologie der Blutgerinnung und der Thrombose. Zieglers Beiträge zur pathologischen Anatomie, Bd. 34 .

Hauser, G. (92): Ein Beitrag zur Lehre von der pathologischen Fibringerinnung. Deutsch. Arch. f. klin. Med. Bd. 50.

Derselbe (98): Über die Bedeutung der sogenannten Gerinnungscentren bei Gerinnung entzündlicher Exsudate und des Blutes. Virch. Arch., Bd. 154.

Derselbe (99): Kurze Bemerkungen zu J. A rnolds Artikel: Über die sogenannten Gerinnungscentren. Centralbl. für allg. Pathologie und path. Anatomie, Bd. 10.

H a y em, G. (78): Recherches sur l'évolution des hématies dans le sang de l'homme et des vertébrés. I. Sang des animaux supérieurs (vertêbrés vivipares). Archives de Physiologie norm. et pathol. Ser. 2, t. 5, ann. 10.

Derselbe (79): Recherches sur l'évolution des hématies dans le sang de l'homme et des vertébrés. II. Sang des vertêbrés à globules rouges nucléés. III. Historique. Ebenda Sér. 2, t. 6, ann. 11.

Derselbe (82): Sur le mécanisme de l'arrêt des hémorrhagies. Comptes rendus hebd. des séances de l'Acad. des Sciences de Paris, t. 9ó, Juillet-Décembre. 
Heinz, R. (01): Blutdegeneration und -regeneration. Zieglers Beiträge zur pathologischen Anatomie, Bd. 29.

Helber, E. (05): Über die Entstehung der Blutplättchen und ihre Beziehungen zu den Spindelzellen. Deutsches Archiv für klinische Medizin, Bd. 82.

Hlava, J. (83): Die Beziehung der Blutplättchen Bizzozeros zur Blutgerinnung und Thrombose. Ein Beitrag zur Histogenese des Fibrins. Arch. f. experim. Pathol. u. Pharm. Bd. 17.

Kopsch, Fr. (01): Die Thrombocyten (Blutplättchen) des Menschenblutes und ihre Veränderungen bei der Blutgerinnung. Anat. Anz., Bd. 19;

Knoll, $\mathrm{Ph}$. (96): Über die Blutkörperchen bei wechselwarmen Wirbeltieren. Sitzungsber. d. kais. Akad. d. Wiss. in Wien. Math.-naturw. Kl., Bd. 105 , Abt. 3.

L aker, C. (82): Studien über die Blutseheibchen und den angeblichen Zerfall der weißen Blutkörperchen bei der Blutgerinnung. Sitzungsber. d. k. Akad. d. Wissensch. Bd. 86, Abt. 3.

Derselbe (84): Die ersten Gerinnungserscheinungen - des Säugetierblutes unter dem Mikroskope. Sitzungsber. d. k. Akad.d. Wissensch. Bd. 90, Abt. 3.

Lö wit, M. (83) : Über die Bildung roter und weißer Blutkörperchen. Sitzungsberichte d. kais. Akademie d. Wissensch., math.-naturw. Cl., Bd. 88, Abt. 3, Jahrgang 1883.

Derselbe (84): Beiträge zur Lehre von der Blutgerinnung. Erste Mitteilung. Über das . coagulative Vermögen der Blutplüttchen. Ebenda Bd. 89, Abt. 3, Jahrgang 188t. - Zweite Mitteilung. Über die Bedeutung der Blutplättchen. Ebenda. Bd.90, Abt. 3, Jahrgang 1884.

Derselbe (85): Über Neubildung nnd Zerfall weißer Blutkörperchen. Ein Beitrag zur Lehre von der Leukaemie. Ebenda, Bd. 92, Abt. 3, Jahrgang 1885 .

Luzet, Ch. (91): Étude sur la régénération du sang après saignée chez les oiseaux. Archives de Physiologie norm. et path. Sér. 5, t. 3, ann. 23.

Macallum, A. B. (92): Studies on the blood of Amphibia. Transactions of the Canadian Institute, vol. 2, 1890-91

Marquis, C. (92): Das Knochienmark der Amphibien in den verschiedenen Jahreszeiten. Inaugural-Dissertation, Dorpat.

Muller, H.F. (89): Zur Frage der Blutbildung. Sitzungsber. der kais. Akad. d. Wissensch. in Wien, math.-naturw. Cl., Bd. 98, Abt. 3.

Neumann, E. (96): Hämatologische Stpudien. Virchows Arch., Bd. 143.

Ranvier, L. (73): Du mode de formation de la fibrine dans le sang extrait des vaisseaux. Comptes rendus des séances de la Soc. de biologie.

D’e rselbe (75): Traité technique d'histologie, Paris.

v. Recklinghausen (66): Übrr die Erzeugung von roten Blutkörperchen. Arch. f. mikr. Anat. Bd. 2.

Riess, L. (04): Über die Beziehungen der Spindelzellen des Kaltblüterblutes zu den Blutplättchen der Säugetiere. Arch. f. exper. Path. u. Pharm., Bd. 51 . 
Schimmelbusch, C. (85): Die Blutplättchen und die Blutgerinnung. Virchows Arch. Bd. 101.

Schmidt, Al. (77): Die Lehre von den fermentativen Gerinnungserscheinungen in den eiweißartigen tierischen Körperflüssigkeiten. Dorpat.

Schultze, M. (65): Ein heizbarer Objekttisch und seine Verwendung bei Untersuchungen des Bluts. Arch. f. mikr. Anat., Bd. 1.

Schwalbe, E. (99): Die morphologischen Umwandlungen der roten Froschblutkörperchen bei der extravasculären Gerinnung. Virchows Arch. $\mathrm{Bd} .158$.

Derselbe (00): Untersuchungen zur Blutgerinnung. Beiträge zur Chemie und Morphologie der Coagulation des Blutes. Braunschweig.

Stricker, S. (77): Beobachtungen über die Entstehung des Zellkernes. Sitzgsber. d. kais. Akademie d. Wiss., math.-naturw. Cl., Bd. 76, Abt. 3.

Vulpia n, A. (77): De la régénération des globules rouges chez les grenouilles à la suite d'hémorrhagies considérables. Compt. rend. hebd. des séances de l'Acad. des Sciences de Paris, t. 84.

Zahn, F. W. (74): Untersuchungen über Thrombose. Virchows Archiv. Bd. 62. Zenker, K. (95)): Über intravasculäre Fibringerinnung bei der Thrombose. Nach dem Tode des Verfassers herausgegeben von Prof. Dr. G. Ha user. Zieglers Beiträge zur pathol. Anatomie, Bd. 17.

Zieler, K. (03): Zur Morphologie des Froschblutes. Nebst Bemerkungen zur "Blutplättchen"frage und zur Entstehung und Bedeutung der radiären Anordnung roter Blutkörperchen um Spindeln (Thrombocyten) und Leukocyten. Pathologisch-anatomische Arbeiten, Orth gewidmet; Berlin.

\section{Erklärung der Abbildungen auf Tafel XXII-XXV.}

Die Abbildungen der Taf. XXII-XXV betreffen morphologische Elemente, hauptsächlich Spindelzellen, des Blutes von Salamandra maculosa. Sie sind sämtlich mit Zeiß' Apochromat $2 \mathrm{~mm}$ (Apertur 1,30) und Kompensationsocular 8 bei Projektion auf Objekttischhöhe gezeichnet.

T'afel XXII.

Spindelzellen aus frischem Blut.

Fig. 1-3. Spindelzellen unmittelbar nach Anfertigung des Präparates; das Protoplasma der in Fig. 2 abgebildeten Zelle enthält Hayem sche Kügelchen.

Fig. 4a-f. Sechs aufeinander folgende Stadien der Veränderung ein- und derselben Spindelzelle; in den ersten zehn Minuten nach Anfertigung des Präparates. Text pag. 329-330. 
Fig. 5-12. Metamorphosierte Spindelzellen, ca. 15 bis 30 Minuten nach Entnahme des Blates. Text pag. 330-331. In Fig. 8 zeigt der dem Cytoplasmaklümpchen benachbarte Teil des Kerns ein eigentümliches helles Aussehen; darin eine netzige Struktur. In Fig. 5, 6, 8, 10 und 12: Ha y em sche Kügelchen.

Fig. 13. Metamorphosierte Spindelzelle, degeneriert. Kern gequollen und von einem hyalinen Hof umgeben. Ca. 20 Minuten nach Anfertigung des Präparats.

Fig. 14. Metamorphosierte Spindelzelle (ca. ${ }^{1 / 2}$ Stunde nach Anfertigung des Präparats) mit zwei dem Cytoplasmaklümpchen angelagerten roten Blutkörperchen, welche deformiert sind.

Fig. 15. Gruppe von zwei metamorphosierten Spindelzellen (ca. 1/2 Stunde nach Anfertigung des Präparates) mit sogenannter Sternanordnung der umgebenden roten Blutkörperchen.

\section{Tafel XXIII.}

Fig. 16. Spindelzelle, fixiert und gefärbt mit dem von $D$ ek hu y z en empfohlenen Gemisch von 1,5\% iger Oșmiumsäure und 1,5\% iger Essigsäure, welches $6 \%$ kalt gesättigte wässerige Methylenblaulösung und etwas Säurefuchsin enthält.

Fig. 17. Spindelzelle aus einem in $1 \%$ iger Sublimatlösung (mit $1 \% 0 \mathrm{NaCl}$ ) fixierten Schleuderpräparat, nach Ehrlich-Bi on di gefärbt.

Fig. 18-26. Metamorphosierte Spindelzellen, nach verschieden langem Aufenthalt in der feuchten Kammer (bei Fig. 18: 2 Minuten, bei Fig. 19: 7 Minuten, bei Fig. 20-26: 15 bis 30 Minuten) in $1 \%$ iger Sublimatlösung (mit 1\% NaCl) fixiert und nach Ehrlich-Bi ondi gefärbt. Die feinen Fäden, welche in Fig. 18, 19, 22-24 von den Zellen ausgehen, sind „dickere" Fibrinfasern; die feine Fibrinstrahlung ist nicht sichtbar. - Der abgeschnürte Protoplasmaballen unten links rom Kern der Fig. 21 gehört zu dieser Figur.

Fig. 27 und 28. Gruppen von zwei und drei metamorphosierten Spindelzellen, 1/2 Stunde nach Anfertigung des Präparates. Fixierung und Färbung wie vorher.

\section{Tafel XXIV und XXV.}

Die Abbildungen der Tafel XXIV und XXV sind nach Präparaten gezeichnet, welche mit $\mathrm{schwachem} \mathrm{Flemmingschen} \mathrm{Gemisch} \mathrm{(mit} 1 \% \mathrm{NaCl}$ ) fixierund hinterher der Flemming schen Dreifachbehandlung (Safranin-GentianaOrange) unterworfen waren.

\section{Tafel XXIV.}

Fig. 29 und 30. Spindelzellen aus einem Schleuderpräparat.

Fig. 31. Aus einem Präparat, welches nach $1 / 2$ stündigem Aufenthalt in der feuchten Kammer fixiert war. Primäre Fibrinmembran. Oben ein rotes Blutkörperchen, in einer Höhle der primären Fibrinmembran liegend; unten ein in Kriechbewegung fixierter Leukocyt, welcher sich einen Gang in der primären Fibrinmembran gebahnt hat. 
Fig. 32-36. Metamorphosierte Spindelzellen. Cytoplasmaklümpchen von feiner Fibrinstrahlung umgeben. Die Zelle der Fig. 32 nach einem Aufenthalt von 2 Minuten, diejenigen der Figg. 33-36 nach einem solchen von 30 Minuten in der feuchten Kammer fixiert. In Fig. 35 und 36 sieht man neben der feinen Fibrinstrahlung dickere Fibrinfasern. In Fig. 35 ist rechts oben ein Ausschnitt der feinen Fibrinstrahlung weggelassen.

Fig. 37. In der Mitte: metamorphosierte Spindelzelle mit feiner Fibrinstrahlung. Oben rechts und oben links je ein rotes Blutkörperchen; unten rechts ein Leukocyt in Kriechbewegung; unten links ein sogenannter „freier Kern". Man konstatiert, daß die Fäden der Fibrinstrahlung $\ddot{u} b$ e $r$ die roten Blutkörperchen und den in Kriechbewegung befindlichen Leukocyten hinweggehen. - Fixiert nach 1/2 stündigem Aufenthalt in der feuchten Kammer.

Fig. 38. Spindelzelle, bei welcher die Metamorphose einen abnormen Verlauf genommen hat. Text pag. 332. - Fixiert nach $1 / 2$ stündigem Aufenthalt in der feuchten Kammer.

\section{Tafel XXV.}

Die Präparate, welche den Figg. 39-42 und 44 zugrunde liegen, sind nach 1/2 stündigen, diejenigen, nach welchen die Figg. 43,45 und 46 gezeichnet.sind, nach zweistündigem Aufenthalt in der feuchten Kamuer fixiert.

Fig. 39. Zwei metamorphosierte Spindelzellen, jede von einer feinen Fibrinstrahlung umgeben; zwischen ihnen eine Spindelfigur.

Fig. 40. Metamorphosierte Spindelzelle mit feiner Fibrinstrahlung. Netz von dickeren Fibrinfasern, welehe in der Nachbarschaft der Spindelzelle radiär :um diese herum angeordnet sind.

Fig. 41. Ein von einer Spindelzelle abgetrenntes Cytoplasmastứck, metamorphosiert, von feiner Fibrinstrahlung umgeben.

Fig. 42. Metamorphosierte Spindelzelle, degeneriert; Kern gequollèn und von einem hyalinen Hof umgeben; wie in Fig. 13.

Fig. 43-45. Gruppen von je zwei Spindelzellen, Fig. 46 größerer Haufen von solchen, umgeben von mehr oder weniger stark deformierten roten Blutkörperchen. In Fig. 44 rechts unten ein Leukocyt (teilweise). 
Archiv tmikroskop.Anatomie. Bd:LxVIII.

Taf.XXIII.

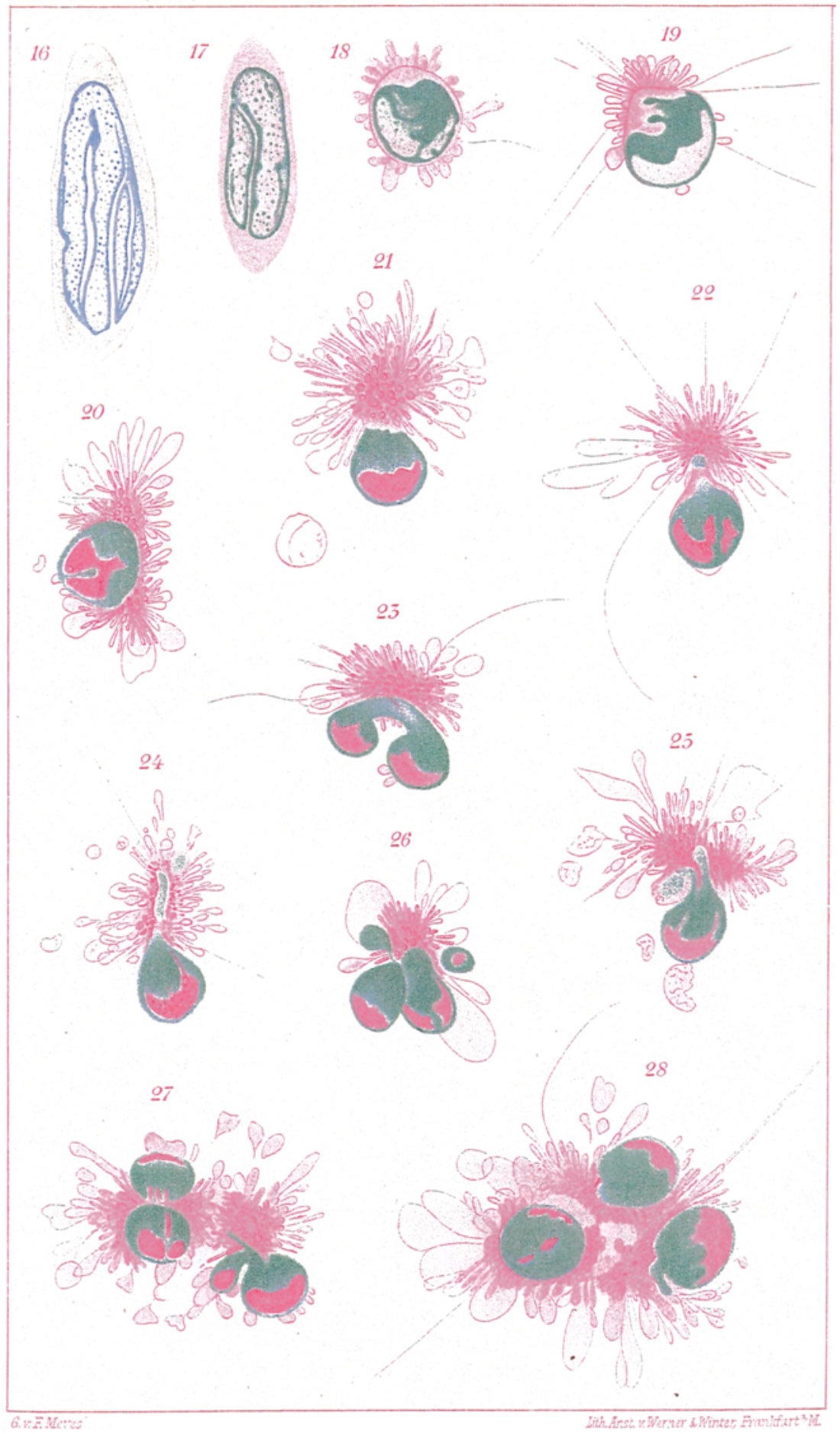

